\title{
The chemical composition of the Sun
}

\author{
MARTin ASPLUnd \\ Max-Planck-Institut für Astrophysik, D-85741 Garching, Germany; e-mail: \\ asplund@mpa-garching.mpg.de \\ NiCOLAS GREVESSE \\ Centre Spatial de Liège, Université de Liège, B-4031 Angleur-Liège, Belgium \\ Institut d'Astrophysique et de Géophysique, Université de Liège, B-4000 Liège, \\ Belgium; e-mail: Nicolas.Grevesse@ulg.ac.be
}

\author{
A. Jacques Sauval \\ Observatoire Royal de Belgique, B-1180 Bruxelles, Belgium; e-mail: \\ jacques.sauval@oma.be
}

\begin{abstract}
PAT SCOTT
Department of Physics and Oskar Klein Centre for Cosmoparticle Physics, Stockholm University, AlbaNova University Centre, S-10691 Stockholm, Sweden; e-mail: pat@fysik.su.se
\end{abstract}

Key Words Sun, solar abundances, meteoritic abundances, solar atmosphere, spectral line formation, convection

\begin{abstract}
The solar chemical composition is an important ingredient in our understanding of the formation, structure and evolution of both the Sun and our solar system. Furthermore, it is an essential reference standard against which the elemental contents of other astronomical objects are compared. In this review we evaluate the current understanding of the solar photospheric composition. In particular, we present a redetermination of the abundances of nearly all available elements, using a realistic new 3-dimensional (3D), time-dependent hydrodynamical model of the solar atmosphere. We have carefully considered the atomic input data and selection of spectral lines, and accounted for departures from LTE whenever possible. The end result is a comprehensive and homogeneous compilation of the solar elemental abundances. Particularly noteworthy findings are significantly lower abundances of carbon, nitrogen, oxygen and neon compared with the widely-used values of a decade ago. The new solar chemical composition is supported by a high degree of internal consistency between available abundance indicators, and by agreement with values obtained in the solar neighborhood and from the most pristine meteorites. There is, however, a stark conflict with standard models of the solar interior according to helioseismology, a discrepancy that has yet to find a satisfactory resolution.
\end{abstract}




\section{CONTENTS}

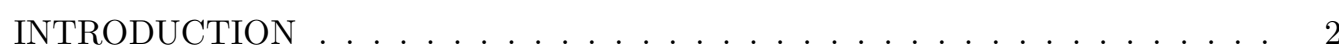

INGREDIENTS FOR SOLAR ABUNDANCE ANALYSIS . . . . . . . . . . 3

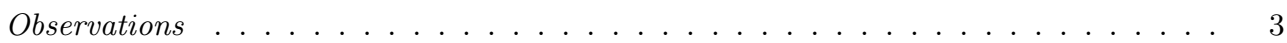

Atomic and molecular data . . . . . . . . . . . . . . . . . . . . 4

Solar atmospheric and line formation modelling . . . . . . . . . . . . . . . . 5

Observational constraints on solar modelling . . . . . . . . . . . . . . . . . 7

PHOTOSPHERIC ABUNDANCES .................... 8

Lithium, Beryllium and Boron . . . . . . . . . . . . . . . . . . . . . . . . . . . . . . . . . . . . . . . .

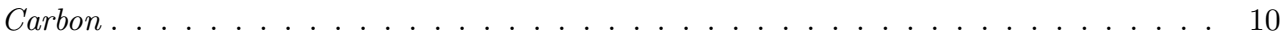

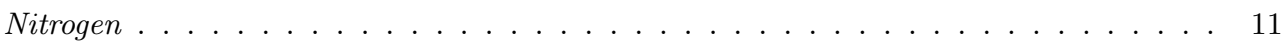

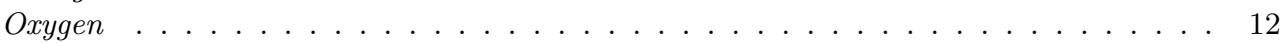

Intermediate Mass Elements . . . . . . . . . . . . . . . . . . . 14

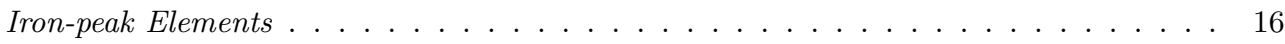

Neutron Capture Elements . . . . . . . . . . . . . . . . . . . 18

Abundances from Sunspots: $\mathrm{F}, \mathrm{Cl}, \mathrm{In}, \mathrm{Tl} \ldots \ldots \ldots \ldots \ldots$

Indirect Determinations: Noble Gases . . . . . . . . . . . . . . . . . . . . . . . . . . . 20

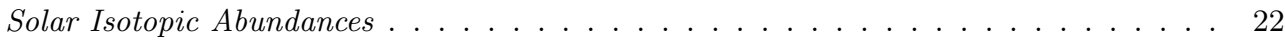

Conversion From Photospheric to Bulk Abundances . . . . . . . . . . . . . . . 23

Solar metallicity . . . . . . . . . . . . . . . . . . . . . . . 24

OTHER METHODS FOR INFERRING SOLAR ABUNDANCES . . . . . . . 24

Meteorites . . . . . . . . . . . . . . . . . . . . 24

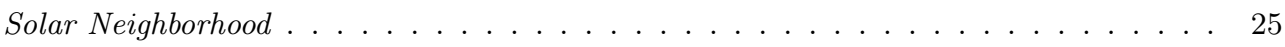

Helioseismology . . . . . . . . . . . . . . . . . . . . . . 27

Solar neutrinos . . . . . . . . . . . . . . . . . . . . . . . . . 29

CONCLUDING REMARKS ....................... . . 30

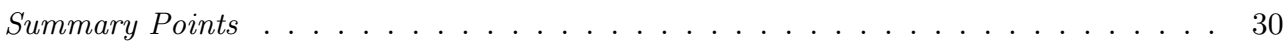

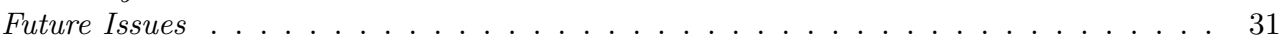

Disclosure Statement . . . . . . . . . . . . . . . . . . . . . . . . . . . . . . . . . . . .

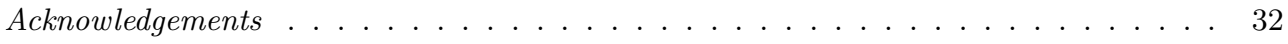

\section{INTRODUCTION}

The solar chemical composition is a fundamental yardstick in astronomy, to which the elemental abundances of essentially all cosmic objects, be they planets, stars, nebulae or galaxies, are anchored. The importance of having accurate solar elemental abundances can thus not be overstated. From the pioneering efforts of Russell (1929), Suess \& Urev (1956) and Goldberg. Müller \& Aller (1960) to the more recent works of Anders \& Grevesse (1989), Grevesse \& Sauval (1998), Lodders (2003), Asplund. Grevesse \& Sauval (2005), Grevesse, Asplund \& Sauval (2007) and Lodders, Palme \& Gail (2009) compilations of the solar system abundances have found extremely wide-ranging use in astronomy and cosmology.

There are two independent and complementary ways of determining the solar system abundances, each with its pros and cons. Through mass spectroscopy of meteorites in terrestrial laboratories, it is possible to directly measure the abundance of almost every element and isotope with remarkable precision. The most pristine meteorites are the so-called CI chondrites, which have been modified least by various physical and chemical processes over the past $4.56 \mathrm{Gyr}$. Even in these meteorites volatile elements have been depleted to various degrees, including 
the six most abundant elements: hydrogen, helium, carbon, nitrogen, oxygen and neon. As a consequence, it is not possible to rely on meteorites to determine the primordial solar system abundances for such elements. This also implies that one must measure all meteoritic abundances relative to an element other than hydrogen, traditionally chosen to be silicon. Prior knowledge of the solar photospheric Si abundance is therefore required in order to place the meteoritic abundances on the same absolute scale as the Sun (Suess \& Urey 1956).

With the exception of a general $\sim 10 \%$ modification due to diffusion and gravitational settling (Sect. 3.11) and depletion of lithium and possibly beryllium, today's photospheric abundances are believed to reflect those at the birth of the solar system. They are of profound importance for the understanding of our own solar system and the Sun's interior structure and evolution. Unfortunately, photospheric abundances cannot be determined with the same accuracy as those from meteorites. In particular, very little isotopic abundance information is available. Furthermore, photospheric abundances are not observed but inferred. Thus the solar spectrum must be interpreted using realistic models of the solar atmosphere and spectrum formation process. In addition, the required atomic and molecular data are rarely precise enough to rival the accuracy of meteoritic measurements. In spite of the remaining uncertainties in solar photospheric determinations, having access to the most accurate solar abundances possible is crucial, not only for understanding our own Sun but also in order to make meaningful comparisons with other stars (for which meteoritic information is obviously unavailable). The Sun is also the only possible reference for the interstellar medium, H II regions and other galaxies when studying the elements depleted in meteorites.

In the decade since the appearance of the widely-used compilation of the solar chemical composition by Grevesse \& Sauval (1998), the abundances of a large number of elements have been revised, several rather severely (e.g. Asplund. Grevesse \& Sauval 2005). This is true in particular for C, N, O and Ne. There are three main reasons for the changes: improvements in atomic and molecular transition probabilities, the advent of 3D hydrodynamical solar model atmospheres and relaxation of the assumption of local thermodynamic equilibrium in the spectral line formation. Several of the proposed abundance alterations have not gone unchallenged, however. It is therefore timely to now critically assess the reference solar chemical composition and make recommendations regarding the most accurate values available to the astronomical community. After recapitulating the necessary ingredients in any solar abundance analysis (Sect. 2), we discuss in detail the solar chemical composition on an element-by-element basis (Sect. 3). We also present a comparison between photospheric solar abundances and those inferred by alternative means, such as via meteorites and stars or gas in the solar neighborhood (Sect. 4). We conclude by summarizing the main points and outlining a few open issues for the future (Sect. [5).

\section{INGREDIENTS FOR SOLAR ABUNDANCE ANALYSIS}

\subsection{Observations}

Analyses of the solar photospheric chemical composition make use of the elemental fingerprints present in the solar spectrum in the form of spectral absorption lines. There are several solar atlases of both intensity and flux spectra in the ultraviolet, optical and infrared regions. The most commonly used opti- 
cal disk-center intensity spectra of the quiet Sun are the so-called Jungfraujoch or Liège atlas (after its locations of observation and production, respectively; Delbouille, Roland \& Neven 1973) and the Kitt Peak solar atlas (Neckel \& Labs 1984). The spectral resolving power of the latter is slightly higher while the former is less affected by telluric absorption due to the higher observing altitude. There are also corresponding infrared disk-center intensity atlases observed from Kitt Peak (Delbouille et al. 1981) and from the ATMOs experiment flown on the space shuttle (Abrams et al. 1996, Farmer \& Norton 1989). Finally, the solar flux spectrum has recently been re-reduced by Kurucz (2006); see also Kurucz et al. (1984) for an earlier rendition of the same Kitt Peak solar data set. All solar atlases agree very well with each other except for spectral regions afflicted by telluric features. Thus the quality of observations is in general not a source of significant error in solar abundance analyses.

\subsection{Atomic and molecular data}

A critical ingredient in any solar abundance analysis is of course the atomic and molecular line input data. Of these the transition probability is the most obvious, but there are a raft of other required components that are not always as well-determined as one would hope. These include line broadening, hyperfine and isotopic splitting, dissociation energies and partition functions. Much laudable work has been done in improving the $g f$-values for a large number of transitions (e.g. Johansson et al. 2003, Kurucz 1992, Lawler et al. 2006, Sobeck, Lawler \& Sneden 2007) but still several elements and lines have surprisingly uncertain transition probabilities. Another important advancement in recent years is that it is now possible to accurately compute the self-broadening (also known as van der Waals broadening) of metal lines (Anstee \& O'Mara 1995, Barklem \& Aspelund-Johansson 2005, Barklem, Piskunov \& O'Mara 2000a), which has made the classical Unsöld broadening and its ad-hoc enhancement factors largely obsolete, at least for neutral species.

The need for atomic data increases dramatically when relaxing the LTE assumption. In non-LTE calculations one needs transition data not only for the line in question but in principle for all other lines of the element. In addition, photo-ionization cross-sections play a crucial role. There have been noteworthy improvements in this respect in recent years through the Opacity and Iron Projects (Badnell et al. 2005) for example, but unfortunately the lack of quantum mechanical calculations for most elements preclude detailed non-LTE investigations. The most unsatisfactory situation, however, relates to collisional cross-sections for excitation and ionization, for which laboratory or theoretical calculations are completely missing bar for a few elements and transitions relevant for spectroscopy of late-type stars (e.g. Barklem 2007a, Belyaev \& Barklem 2003). In the absence of realistic evaluations, classical recipes such as those of van Regemorter (1962) and Drawin (1968) are frequently used but cannot be expected to be more than order-of-magnitude estimates at best. Often scaling factors to these formulae are used, sometimes calibrated by achieving an optimal overall fit to the various spectral features. It should remembered that such a procedure may reveal less about the true collisional cross-sections than about other shortcomings in the modelling of the stellar atmospheres and line formation (Asplund 2005). 


\subsection{Solar atmospheric and line formation modelling}

Extracting information about the Sun's chemical composition from the solar spectrum requires realistic models of the solar atmosphere and the line formation process. Traditional solar and stellar abundance analyses employ one-dimensional (1D) model atmospheres assuming time-independence and hydrostatic equilibrium. Such models come in two flavours: theoretical and semi-empirical. The former are constructed assuming a constant total (radiative plus convective) energy flux through the atmosphere, which dictates the temperature stratification given the adopted equation-of-state and opacities. Convective energy transport is normally described by the mixing length theory (Böhm-Vitense 1958). The radiative flux is determined by solving the radiative transfer equation, typically under the simplification of local thermodynamic equilibrium (LTE). To achieve a realistic temperature structure, treatment of the combined effects of spectral lines ('line blanketing') must be as complete as possible. The most widely-used theoretical 1D model atmospheres for solar-type stars are the Kurucz (1993) and MARCS (Gustafsson et al. 2008) grids of models.

Semi-empirical models are often the preferred choice for solar spectroscopists. In these models, temperatures are inferred from depth-dependent observations such as the center-to-limb variation of the continuum and/or lines with differing formation heights. The resulting atmospheric structure is thus sensitive to the ingredients involved in the inversion process, such as LTE for the continuum and line formation, and the adopted line data. Hydrostatic equilibrium is still assumed in semi-empirical models, but the flux constancy criterion does not need to be obeyed nor is it necessary to estimate convective energy transport. Several semi-empirical solar models are on the market, including the Holweger \& Müller (1974), vAL3C (Vernazza, Avrett \& Loeser 1976) and MISS (Allende Prieto et al. 2001) models. The favoured model of solar abundance aficionados is the Holweger \& Müller model, partially because its temperature stratification is expected to be an accurate representation of the real photosphere - but also partially just by habit. Indeed it is somewhat surprising that the VAL3C model or its successors (e.g. Fontenla et al. 2006) have not caught on more within the solar abundance community, since they are built on similar premises but without the LTE restriction. The venerable Holweger \& Müller model in fact dates back more than forty years, since the 1974 version was simply a new pressureintegration of the original Holweger (1967) model using updated equation-of-state and continuous opacities. It was constructed to match the observed continuum center-to-limb variation as well as the line depths of some 900 lines of varying strengths, under the assumption of LTE. Due to the limited spectral resolution of the solar spectrum used by Holweger, one would expect the temperatures to be overestimated by about $50 \mathrm{~K}$ in the line-forming region. Interestingly, the corrected version of such a temperature structure would be more similar to the MISS semi-empirical model (Allende Prieto et al. 2001); both semi-empirical models have a shallower temperature gradient than 1D theoretical model atmospheres, as seen in Fig. 1. It should be borne in mind that there exists an ambiguity when employing any 1D model: while many simply adopt temperatures, pressures and densities directly from the published model, a more correct procedure is to perform a pressure-integration using only the temperature structure as a function of continuum optical depth, in order to be consistent with the opacities computed with the spectrum synthesis code one employs. 
More recently it has become tractable to perform 3D, time-dependent, hydrodynamical simulations of the stellar surface convection (e.g. Asplund et al. 1999, Frevtag, Steffen \& Dorch 2002, Stein \& Nordlund 1998, Vögler, Bruls \& Schüssler 2004). Here the standard conservation equations of mass, momentum and energy are solved together with the $3 \mathrm{D}$ radiative transfer equation in a small but representative volume of the stellar atmosphere. Radiative energy exchange with the gas in the surface layers is crucial, because this is what drives the convective motions. Because of the much more computationally demanding nature of such hydrodynamical modelling, approximations must be made in the radiative transfer treatment. Rather than solving for many thousands of frequency points as regularly done in theoretical 1D hydrostatic models, the wavelength variation of opacity is represented by a small number of typical opacity bins (4-20) in order to compute the radiative heating/cooling rate that enters the energy equation (Nordlund 1982). Detailed comparisons with the monochromatic solution reveal that this scheme is surprisingly accurate. The reader is referred to Nordlund. Stein \& Asplund (2009) for a recent review of the physics of solar surface convection and the numerical details of its simulation.

A few different 3D hydrodynamical solar model atmospheres have been constructed and employed for abundance purposes (e.g. Asplund et al. 2000b, Caffau et al. 2008a, Trampedach et al. 2009). While these are computed with different codes, they are based on many of the same physical assumptions and approximations. It is therefore not too surprising that the resulting atmospheric structures are similar. The 3D models have been computed using realistic and comprehensive equation-of-state, continuous and line opacities. The numerical resolution is sufficiently high to adequately resolve the granulation, albeit obviously not high enough to reach down to the microscopic viscous dissipation length-scales. The reason that these so-called 'large-eddy' simulations are nevertheless quite successful in reproducing the real photosphere is that the mean stratification is largely independent of numerical resolution Asplund et al. 2000a, Stein \& Nordlund 1998). Spectral line formation is heavily biased to the upflows, which have a low degree of turbulence in comparison to the downflows, due to the divergent flow. Obviously none of the mixing length parameters required in 1D models enter into 3D simulations, as the convective energy flux is instead a natural consequence of the hydrodynamics.

Having a suitably realistic solar model atmosphere, the next step when trying to infer the solar chemical composition is to model how the spectrum is formed. In order to derive an abundance of an element, solar spectroscopists either measure the total observed line strength as defined by the equivalent width, or directly compare the observed and theoretical spectra through the fitting of line profiles. Finally, to compute the line strength requires knowledge of the level populations. In LTE, these follow straightforwardly from the local temperature and electron pressure using the Boltzmann and Saha distributions. In general this rather severe simplification cannot be expected to be valid, and the non-LTE case must instead be considered. While non-LTE is a general term covering all situations when LTE is not valid, in practice all calculations referred to herein assume statistical equilibrium, i.e. the level populations do not change with time. NonLTE thus requires a simultaneous solution of the rate equations for all relevant levels and species together with the radiative transfer equation for all relevant wavelengths. We refer to Asplund (2005) for a detailed account of the principles of non-LTE and the most important non-LTE effects encountered for late-type 
stars like the Sun.

\subsection{Observational constraints on solar modelling}

Since more sophisticated modelling does not automatically imply a more realistic outcome, it is of paramount importance to carefully test the predictions of the 3D model atmospheres against an arsenal of observational diagnostics. This is especially true given that 1D model atmospheres more often than not fail the very same tests. Current generations of 3D models are very successful in reproducing the observed solar granulation topology, typical length- and time-scales, convective velocities and intensity brightness contrast (e.g. Nordlund, Stein \& Asplund 2009, Stein \& Nordlund 1998), clearly none of which 1D models are able to predict.

Another important constraint is the center-to-limb variation of the continuum as a function of wavelength. Avres. Plvmate \& Keller (2006) have criticized the 3D hydrodynamical model of Asplund et al. (2000b), oft used in recent solar abundance analyses, for having too steep a temperature gradient as judged by the observed center-to-limb variation. Koesterke. Allende Prieto \& Lambert (2008) reach a somewhat milder conclusion, presumably because they performed spectral synthesis with the full 3D model rather than with only the spatiallyaveraged version used by Ayres, Plymate \& Keller. The 3D CO5BOLD solar model of Caffau et al. (2008a) appears to perform better in this respect (H. Ludwig, private communication). In the present work, we make extensive use of the 3D solar model by Trampedach et al. (2009), which has been constructed with an improved treatment of the radiative transfer and updated opacities. Because of its somewhat shallower temperature stratification than the $3 \mathrm{D}$ model of Asplund et al. (Fig. 11), the new 3D model satisfies the center-to-limb variation constraint very well, as illustrated in Fig. 2. Indeed it outperforms all 1D models, even the semi-empirical (Holweger \& Müller 1974) model, which was designed to fit this diagnostic (Pereira et al. 2009).

The wings of the hydrogen Balmer lines are also sensitive tracers of the temperature stratification in the deeper layers of the photosphere. In recent years, significant improvements have been made in the treatment of the broadening of $\mathrm{H}$ lines, especially self-broadening (Allard et al. 2008; Barklem, Piskunov \& O'Mara 2000b). It is still unclear whether noticeable non-LTE effects can be expected in the solar atmosphere, since available calculations for the cross-sections of inelastic $\mathrm{H}+\mathrm{H}$ collisions are uncertain (Barklem 2007b). With 1D theoretical model atmospheres, the $\mathrm{H}$ lines imply too low $T_{\text {eff }}$ for the Sun by $50-100 \mathrm{~K}$, and have the wrong line shapes when the most accurate line broadening data are used (Barklem et al. 2002). Such problems can be partly cured by playing with the mixing length parameters, however. In contrast, the $\mathrm{H}$ lines suggest that the temperature gradient of the Holweger \& Müller (1974) semi-empirical model is too shallow. Pereira et al. (2009) found that the 3D solar model of Trampedach et al. (2009) reproduces the $\mathrm{H} \alpha$ and $\mathrm{H} \beta$ lines very well in LTE, doing so significantly better than the 1D models they investigated, but without requiring the tweaking of any free parameters. The CO5BOLD model has to our knowledge not yet been tested against $\mathrm{H}$ lines.

As seen in Fig. 3, with 3D hydrodynamical models for the solar atmosphere it is now possible to achieve highly satisfactory agreement with observed profiles of typical weak and intermediate strong lines (the wings of strong lines reveal more 
about the pressure broadening data than the employed model atmosphere). It is important to remember that this is achieved without invoking the free parameters necessary in any 1D analysis (micro- and macroturbulence) in the line formation calculations. Such line broadening can thus be explained as the result of the Doppler shift arising from the convective motions with a smaller contribution from the solar oscillations (e.g Nordlund, Stein \& Asplund 2009, and references therein). Indeed, even the observed line shifts and asymmetries are very well reproduced with 3D models (e.g. Asplund et al. 2000b). The CO5BOLD solar model produces good overall line profiles (e.g. Caffau et al. 2008a) but whether this extends also to line asymmetries has not yet been investigated.

In summary, the 3D hydrodynamical model atmosphere (Trampedach et al. 2009) that we draw results from in this review outperforms 1D model atmospheres in the available observational tests, from granulation topology and center-to-limb variation to $\mathrm{H}$ lines and the detailed profiles of metallic lines. For the purposes of photospheric abundance determinations, the 3D solar model employed here appears to be a very realistic representation of the solar photosphere. No large differences are expected from using our 3D model or the corresponding CO5BOLD model (Caffau et al. 2008a) given their overall similarities.

\section{PHOTOSPHERIC ABUNDANCES}

Our recommended solar photospheric elemental abundances are listed in Table 1. which also provides the corresponding meteoritic values for CI chondrites to be discussed in Sect. 4.1. [Throughout this review, we adopt the customary astronomical scale for logarithmic abundances where hydrogen is defined to be $\log \epsilon_{\mathrm{H}}=12.00$, i.e. $\log \epsilon_{\mathrm{X}}=\log \left(N_{\mathrm{X}} / N_{\mathrm{H}}\right)+12$, where $N_{\mathrm{X}}$ and $N_{\mathrm{H}}$ are the number densities of element $\mathrm{X}$ and hydrogen, respectively.] Fig. 4 shows how the solar abundances vary with atomic number, illustrating several key features of nuclear and stellar physics: the high primordial $\mathrm{H}$ and He abundances; the fragile nature of $\mathrm{Li}, \mathrm{Be}$ and $\mathrm{B}$; the relatively high abundance of elements involved in stellar $\mathrm{H}-$, He- and C-burning, modulated by the odd-even effect and the $\alpha$-capture effect; the high nuclear binding energy and near nuclear statistical equilibrium for the Fe-peak; and the production of the heavy elements through successive neutron capture with peaks around the magic nuclei possessing closed neutron shells (e.g. Pagel 1997).

In this review, we have attempted to reanalyse the solar abundances of (nearly) all elements in a homogeneous manner with the best possible atomic data and state-of-the-art solar modelling. As a guiding principle, we have been very discerning when selecting the lines for each element, since inclusion of dubious lines only increases the abundance scatter and tends to skew the results towards higher abundances due to blends. The analysis has been carried out using several 1D and 3D model atmospheres and with non-LTE effects accounted for whenever possible, all done with the same well-tested computer codes. Unless specified otherwise, the results presented here have been based on the 3D hydrodynamical solar model atmosphere of Trampedach et al. (2009). Below we present in some detail how the abundances were derived but the full description of the analysis (including line lists with all the relevant data for the transitions) will appear in a forthcoming series of articles in Astronomy 83 Astrophysics (Asplund et al. 2009a.,b.c, Grevesse et al. 2009, Sauval et al. 2009, Scott et al. 2009b). 
There are a wide range of potential sources of error in solar and stellar abundance analyses, from inaccurate input data for the transition $(g f$-values, line broadening etc) to difficulties in estimating line strengths (finite $S / N$, continuum placement, blends etc), to inadequacies in the atmospheric and line-formation modelling (1D, LTE, mixing length and microturbulence parameters, continuous opacities, etc). No consensus exists in the solar abundance literature on how the uncertainties should be quantified. Some authors have used the standard deviation arising from the chosen sample of lines to represent the total error for a given species whilst others have employed the standard error of the mean or some different measure altogether. Previous compilations of the solar chemical composition contained a mixture of different error estimates, since the values were taken from sources with differing modus operandi.

We have attempted to quantify three possible systematic errors introduced by the modelling: mean atmospheric stratification, atmospheric inhomogeneities and departures from LTE. The first uncertainty has been estimated by taking half the difference between the results with the spatially-averaged 3D model (denoted $<3 \mathrm{D}>$ ) and the Holweger \& Müller (1974) model. The second has been evaluated as half the difference between the full $3 \mathrm{D}$ results and those from $<3 \mathrm{D}>$. The inclusion of the theoretical MARCS (Gustafsson et al. 2008) and semi-empirical MISS (Allende Prieto et al. 2001) models serves as a further check on these two systematic errors. Due to the general lack of non-LTE calculations for the majority of elements, the non-LTE uncertainty is more difficult to quantify. Somewhat arbitrarily, in most cases we have chosen half of the predicted non-LTE abundance correction as an estimate of the error. Occasionally we have employed the difference between the cases with and without inelastic $\mathrm{H}$ collisions through the Drawin (1968) formula, whilst taking into account the recommendations by Asplund (2005). We have used an arbitrary, minimal estimate of the possible errors in the non-LTE corrections of 0.03 dex. In some cases this might overestimate the non-LTE systematic error, whilst in others (e.g. TiI) it probably underestimates the error; there is unfortunately no other consistent way to include such unknown errors. Estimating possible systematic errors arising from inappropriate line input data has not been attempted here; relative errors in $g f$-values will at least be accounted for in the dispersion of the lines. When the elemental abundance is determined by only one or very few lines, we have attempted to estimate an additional error arising from uncertainties due to continuum placement and possible blends. The systematic errors have been added in quadrature with the statistical error to estimate the total uncertainty, which is used throughout the article unless specified otherwise. The statistical error has been computed from the weighted standard error of the mean, with weights assigned to each line according to uncertainties in the continuum placement and known or suspected minor blends.

\subsection{Lithium, Beryllium and Boron}

Lithium: In the Sun, Li is depleted by about a factor of 150 compared with the already small meteoritic value. Even the Li I resonance line at $670.8 \mathrm{~nm}$ is therefore very weak. The line is blended by an assortment of CN and Fe I lines, which makes the $\mathrm{Li}$ abundance determination very challenging. Because the transition arises from the ground state of a minority species, the line is sensitive to both the temperature and photo-ionizing UV radiation field Asplund 2005, 
Kiselman 1997). The recommended value given in Table 1 stems from the analysis of Müller, Peytremann \& de La Reza (1975), which has been corrected for the effects of atmospheric inhomogeneities and 3D non-LTE effects (Asplund et al. 1999, Barklem, Belyaev \& Asplund 2003). The solar Li depletion cannot be explained by standard models based on the mixing length theory of convection but requires additional mixing below the convection zone (Brun, Turck-Chièze \& Zahn 1999; Charbonnel \& Talon 2005).

Beryllium: Early work implied that Be was depleted in the solar convection zone by about a factor of two (Chmielewski. Brault \& Müller 1975). This interpretation was challenged by Balachandran \& Bell (1998), who argued that one must take into account the long-suspected missing UV opacity (e.g. Short \& Hauschildt 2005, and references therein) in synthesizing the Be II resonance lines at $313 \mathrm{~nm}$. They estimated the amount of missing opacity by requiring that the nearby $\mathrm{OH}$ electronic lines would return the same $\mathrm{O}$ abundance as the $\mathrm{OH}$ vibrational lines in the infrared, which increased the derived photospheric Be abundance to the meteoritic value. The required extra opacity can be largely attributed to photoionization of Fe I (Bell. Balachandran \& Bautista 2001). The Balachandran \& Bell analysis was corroborated by Asplund (2004), who found that the basic conclusions are independent of the adopted model atmosphere, whether 1D or 3D. The Be II lines are not sensitive to non-LTE effects in the Sun (Asplund 2005).

Boron: The problem of the missing UV opacity may also impact the solar $\mathrm{B}$ abundance determination, which can realistically only be based on the B I $249.7 \mathrm{~nm}$ resonance line. Cunha \& Smith (1999) have re-analysed the line with careful consideration of the important $\mathrm{Mg}$ I photo-ionization cross-sections. The effects of departures from LTE (Kiselman \& Carlsson 1996) and 3D hydrodynamical model atmospheres (Asplund. Grevesse \& Sauval 2005) are small and opposite in sign. There is still a large uncertainty attached to the resulting abundance, $\log \epsilon_{\mathrm{B}}=2.70 \pm 0.20$, due to the difficulty in analysing this crowded spectral region. Not surprisingly, B does not appear depleted in the solar photosphere.

\subsection{Carbon}

The solar carbon abundance can be inferred from a wealth of indicators, from lowexcitation forbidden and high-excitation permitted atomic lines to various molecular transitions of CH, $\mathrm{C}_{2}$ and $\mathrm{CO}$ (Lambert 1978). This fortunate circumstance has not translated into a well-determined abundance over the years as the different diagnostics have often yielded discrepant results. The preliminary abundance $\log \epsilon_{\mathrm{C}}=8.56 \pm 0.04$ recommended by Anders \& Grevesse (1989) eventually became $8.60 \pm 0.05$ in the final analysis of Grevesse et al. (1991). Grevesse \& Sauval (1998) based their value of $8.52 \pm 0.06$ on an unpublished analysis employing a Holweger \& Müller (1974) model with a modified temperature structure, in an attempt to remove existing trends with excitation potential and equivalent widths for $\mathrm{C}, \mathrm{N}, \mathrm{O}$ and $\mathrm{Fe}$. Over the past decade the solar $\mathrm{C}$ abundance has undergone an even more drastic downward revision. Allende Prieto, Lambert \& Asplund (2002) reanalysed the [C I] $872.7 \mathrm{~nm}$ line and found that the combination of a 3D hydrodynamical solar model (Asplund et al. 2000b) and an updated $g f$-value brought the abundance down to $8.39 \pm 0.04$. The same value was obtained by Asplund et al. (2005), who also employed $\mathrm{CI}, \mathrm{C}_{2}$ and $\mathrm{CH}$ electronic and vibration lines using the same 3D model. Convincingly, all indicators gave consistent results, further strengthening the case for the reality of the low $\mathrm{C}$ abundance. 
In sharp contrast, the abundances estimated with the Holweger \& Müller (1974) model atmosphere disagreed by $\approx 0.2 \mathrm{dex}$. The presence of temperature inhomogeneities and a cooler mean temperature structure in the 3D model compared with the previously employed Holweger \& Müller (1974) model reduced the deduced abundance from the molecular lines in particular, while significant departures from LTE had a similar effect on the C I lines. Using weak lines of the extremely temperature-sensitive $\mathrm{CO}$ molecule and a self-consistently derived $\mathrm{O}$ abundance, Scott et al. (2006) corroborated this finding. They also determined an isotopic ratio of ${ }^{12} \mathrm{C} /{ }^{13} \mathrm{C}=86.8 \pm 3.8$.

The value we recommend here, $\log \epsilon_{\mathrm{C}}=8.43 \pm 0.05$, is slightly higher than in Asplund. Grevesse \& Sauval (2005) and stems from the mean of the 3D-based results for [CI], C I, CH and $\mathrm{C}_{2}$ lines (Asplund et al. 2009a). As illustrated in Table 2, the various $\mathrm{C}$ indicators imply highly uniform abundances. With the exception of a correlation between derived abundance and line strength for $\mathrm{CI}$, there are no trends with transition properties such as excitation potential or equivalent width for any of the species. A similar trend was also present in Asplund et al. (2005), which was attributed to underestimated non-LTE effects for the stronger lines (Fabbian et al. 2006). We note that only 1D non-LTE abundance corrections have been employed for the C I results while the departures from LTE are expected to be exacerbated in the presence of atmospheric inhomogeneities (Asplund 2005); a full 3D non-LTE study for C would clearly be worthwhile. Given the importance of this abundant element, it is essential that the results of Asplund et al. (2009a) are confirmed by an independent group using an independent 3D model, akin to the analysis of the solar $\mathrm{O}$ abundance (Caffau et al. 2008a).

\subsection{Nitrogen}

The nitrogen abundance can be determined from both atomic and molecular lines, although the forbidden [NI] lines are too weak in the solar spectrum to be measurable (Grevesse et al. 1990). A preliminary $\mathrm{N}$ abundance was given in Asplund, Grevesse \& Sauval (2005) using high excitation N I lines and NH vibration-rotation lines in the infrared while in the final analysis (Sauval et al. 2009) also NH pure rotation lines and a multitude of transitions from various bands of $\mathrm{CN}$ were considered in the framework of an improved 3D model. The non-LTE effects for $\mathrm{N}_{\mathrm{I}}$ are relatively small: $\approx-0.05$ dex when ignoring $\mathrm{H}$ collisions (Caffau et al. 2009). In their own analysis of these very weak N I lines using their CO5BOLD 3D solar model, Caffau et al. (2009) obtained a N I-based abundance which is 0.05 dex higher than the corresponding value in Sauval et al. (2009), largely due to the selection of lines. They included many lines that we consider to be of dubious value due to known or suspected blends. This is also reflected in the dramatically different standard deviations for the $\mathrm{N}$ I lines in the two studies: $\sigma=0.04$ and $0.12 \mathrm{dex}$, respectively. Sauval et al. (2009) found very good agreement between abundances inferred from N I and NH lines, with very small dispersion in the NH results. The different $\mathrm{CN}$ bands also gave very similar results when employing a self-consistently derived $\mathrm{C}$ abundance with the 3D model. Unfortunately, Caffau et al. (2009) did not consider molecular lines and it is therefore not known how well their 3D model performs in achieving consistent results for atoms and molecules. It is sometimes stated in the literature that the molecular lines are more temperature sensitive than atomic lines, but in fact the 
$\mathrm{NH}$ lines are less so than these highly excited N I lines.

The $\mathrm{N}$ abundance from Sauval et al. (2009) that we recommend here, $\log \epsilon_{\mathrm{N}}=$ $7.83 \pm 0.05$, is 0.09 dex lower than suggested by Grevesse \& Sauval (1998) and 0.22 dex lower than in Anders \& Grevesse (1989).

\subsection{Oxygen}

Oxygen is the most abundant element in the cosmos not produced in the Big Bang, and the third most common overall. Oxygen and its isotopes are key tracers of the formation and evolution of planets, stars and galaxies and as such it is one of the most important elements in all of astronomy. Nevertheless, or perhaps consequentially, its abundance often seems to be in dispute with the solar $\mathrm{O}$ abundance being no exception. The favoured solar content has dropped almost precipitously over the past two decades from $\log \epsilon_{\mathrm{O}}=8.93 \pm 0.04$ in Anders \& Grevesse (1989) to 8.66 in Asplund. Grevesse \& Sauval (2005). The value we recommend here, $\log \epsilon_{\mathrm{O}}=8.69 \pm 0.05$, is taken from Asplund et al. (2009c), who presented a 3D-based study of the available diagnostics: forbidden [O I] and permitted $\mathrm{O}$ I transitions as well as vibration-rotation and pure rotation lines of $\mathrm{OH}$.

Allende Prieto, Lambert \& Asplund (2001) reanalysed the [O I] $630 \mathrm{~nm}$ line with a $3 \mathrm{D}$ model atmosphere. Based on the line profile they demonstrated that the line is blended by a Ni line, which was subsequently confirmed experimentally (Johansson et al. 2003). In combination with the 3D model, recognition of the blend led to a substantial lowering of the solar $\mathrm{O}$ abundance compared with the canonical value at the time. Ayres (2008) revisited the $630 \mathrm{~nm}$ line with a single snapshot of another 3D model but found a 0.12 dex higher $\mathrm{O}$ abundance, partly because he allowed the Ni I $g f$-value to vary freely in spite of the new accurate measurement by Johansson et al. (2003). Caffau et al. (2008a) used several snapshots from the same CO5BOLD 3D model but instead fixed the Ni contribution assuming the Ni abundance from Grevesse \& Sauval (1998) thereby confirming the results of Allende Prieto, Lambert \& Asplund (2001) and Asplund et al. (2004). A more self-consistent approach is to first determine the Ni abundance before considering the [O I] line, as done by Scott et al. (2009a) with the same 3D model as employed by Asplund et al. (2004). As explained by Scott et al., it is then possible to very accurately predict the Ni I contribution to the overall line strength, independent of the reference atmospheric model. Asplund et al. (2009c) updated this analysis and found that the effects of the increased Ni I proportion and their shallower model temperature gradient in comparison with Allende Prieto, Lambert \& Asplund (2001) compensate for one another, leaving the abundance at $\log \epsilon_{\mathrm{O}}=8.66$. This abundance is also consistent with the centerto-limb variation of the $630 \mathrm{~nm}$ feature (Pereira, Asplund \& Kiselman 2009).

In addition to the $630 \mathrm{~nm}$ line, there are two other [O I] transitions that can be utilized. The $636 \mathrm{~nm}$ line is located in the midst of a very broad Ca I autoionization line as well as blended by CN lines; the latter's contribution can however be accurately predicted from other neighboring CN lines. Both Caffau et al. (2008a) and Asplund et al. (2009c) found that this line returns a slightly higher abundance than the $630 \mathrm{~nm}$ line, but still consistent within the uncertainties. Meléndez \& Asplund (2008) added the [O I] $557 \mathrm{~nm}$ line, whose slightly excited lower level makes it largely independent of the model atmosphere. Unfortunately this transition is badly blended by $\mathrm{C}_{2}$ lines, whose contribution Meléndez \& Asplund 
attempted to estimate using nearby $\mathrm{C}_{2}$ lines with the same line strengths and excitation potential. Asplund et al. (2009c) obtained an abundance from the $557 \mathrm{~nm}$ line exactly in between those from the other two forbidden transitions. The three [O I] lines imply a solar $\mathrm{O}$ abundance of $\log \epsilon_{\mathrm{O}}=8.70 \pm 0.05$ when due consideration is given to the blends.

Recently, Centeno \& Socas-Navarro (2008) introduced a novel method to estimate the $\mathrm{O}$ abundance, namely spectropolarimetry of the $[\mathrm{O} \mathrm{I}] 630 \mathrm{~nm}$ line in a sunspot. Because of the different polarization of the [O I] and Ni I lines it is possible to disentangle the Ni I contribution. After consideration of the fraction of $\mathrm{O}$ tied up in $\mathrm{CO}$ in the cool environments of sunspots, they obtained a high $\mathrm{O}$ abundance of $\log \epsilon_{\mathrm{O}}=8.86$. Scott et al. (2009a) have however pointed out that Centeno \& Socas-Navarro applied an outdated $g f$-value for the [O I] line. Furthermore, they showed that when using an improved Ni abundance and an alternative $\mathrm{CO}$ treatment one finds a significantly lower $\mathrm{O}$ abundance: $\log \epsilon_{\mathrm{O}}=8.71$; a further reduction is obtained when employing an alternative sunspot model.

There are a half-dozen or so permitted, high-excitation O I lines useful for solar abundance purposes. Of these the relatively strong $777 \mathrm{~nm}$ triplet lines are arguably the most reliable since the others are either weak or partly blended. The main challenge when employing the $\mathrm{O}$ I lines relates to quantifying the nonLTE effects, which can be significant. The predicted non-LTE abundance corrections are little dependent on the model atmosphere in question, be it $1 \mathrm{D}$ or 3D (Asplund et al. 2004) but they do depend on the adopted collisional crosssections, in particular the inelastic H collisions (e.g. Asplund 2005, Kiselman 1993). Different approaches have been used in solar abundance works to date where some neglect the $\mathrm{H}$ collisions altogether based on the available atomic physics data for other elements while other use the classical Drawin (1968) formula, possibly with a scaling factor $S_{\mathrm{H}}$ that typically varies from 0 to 1 . Holweger (2001) found $\log \epsilon_{\mathrm{O}}=8.71 \pm 0.05$ using the Holweger \& Müller (1974) model with granulation corrections estimated from a 2D solar model, while Asplund et al. (2004) obtained a 0.07 dex lower abundance from a 3D non-LTE study; the main difference, however, between the two studies is the adopted $\mathrm{H}$ collisions $\left(S_{\mathrm{H}}=1\right.$ and 0, respectively). Allende Prieto. Asplund \& Fabiani Bendicho (2004) attempted to calibrate the poorly known collisional efficiency through a comparison of the center-to-limb variation of the $\mathrm{O}$ I $777 \mathrm{~nm}$ line strengths since the relative importance of $\mathrm{H}$ collisions change as $N_{\mathrm{H}} / N_{\mathrm{e}}$ increases with smaller optical depths. They found that $S_{\mathrm{H}}=1$ was a marginally better fit than $S_{\mathrm{H}}=0$ while the LTE case could be ruled out at high significance level. Recently, Pereira, Asplund \& Kiselman (2009) have revisited the issue equipped with better solar observations and tested additional $S_{\mathrm{H}}$ values but have arrived at a similar conclusion, as can be seen in Fig. 5 .

The O I-based abundance we adopt here stems from Asplund et al. (2009c), which is based on full 3D non-LTE calculations with $S_{\mathrm{H}}=1$. The new electron collisional cross-sections computed by Barklem (2007a) have also been adopted (see Fabbian et al. 2009 for an account of the impact of these on other stars). The revised abundance is slightly higher than in Asplund et al. (2004): $\log \epsilon_{\mathrm{O}}=$ $8.69 \pm 0.05$. Using a different 3D model, Caffau et al. (2008a) obtained $\log \epsilon_{\mathrm{O}}=$ $8.73 \pm 0.06$ (unweighted mean) for their disk-center O I lines, in spite of using $S_{\mathrm{H}}=1 / 3$; with $S_{\mathrm{H}}=1$ their result would be 0.02 dex higher. Because they were restricted to $1 \mathrm{D}$ non-LTE calculations, they were unable to carry out line profile fitting as done by Asplund et al. (2009c). They were therefore forced 
to rely on measuring equivalent widths, which were surprisingly large compared to all previous published studies. Reassuringly, when the same input data are adopted (e.g. line strengths, H collisional efficiency), the Caffau et al. (2008a) and Asplund et al. (2009c) analyses are in excellent agreement, which shows that the exact choice of 3D model is of little consequence for the permitted $\mathrm{O}$ I lines.

Grevesse, Sauval \& van Dishoeck (1984) and Sauval et al. (1984) introduced the vibration-rotation and pure rotation lines of $\mathrm{OH}$ as solar $\mathrm{O}$ abundance indicators. In their 3D-based analysis, Asplund et al. (2004) found that the $\mathrm{OH}$ lines implied an $\mathrm{O}$ abundance only slightly lower than the atomic transitions. While the vibration lines showed no trends with excitation potential or line strength, the pure rotation lines did. This is not too surprising, since these strong rotation lines are formed in very high atmospheric layers, which are the most challenging to model realistically due to for example magnetic fields, non-LTE and nonequilibrium chemistry. Asplund et al. (2009c) revisited the $\mathrm{OH}$ lines equipped with a new $3 \mathrm{D}$ model, which yielded $\log \epsilon_{\mathrm{O}}=8.69$ and 8.69 for the vibrationrotation and pure rotation lines, respectively; the trend with line strength for the latter were still present but somewhat reduced and now in the opposite direction. Unfortunately, the $\mathrm{OH}$ lines have not yet been analysed using the alternative 3D model of Caffau et al. (2008a). Meléndez (2004) added a few vibration lines from the first overtone band of $\mathrm{OH}$, which we however do not consider sufficiently reliable abundance indicators due to their extreme weakness.

The 3D-based solar $\mathrm{O}$ abundance that we recommend here, $\log \epsilon_{\mathrm{O}}=8.69 \pm$ 0.05 , is a mean of the [O I], O I, OH vibration-rotation and $\mathrm{OH}$ pure rotation results of Asplund et al. (2009c). The agreement between the different abundance indicators is very satisfactory, which is not the case with the Holweger \& Müller (1974) model as seen in Table2. As explained above, the use of a 3D model is only one factor in the downward revision of the $\mathrm{O}$ abundance from the value $8.83 \pm$ 0.06 given in Grevesse \& Sauval (1998) with refined non-LTE line formation, better accounting of blends and improved atomic and molecular data also playing important roles.

Using CO lines, Scott et al. (2006) determined an isotopic abundance of ${ }^{16} \mathrm{O} /{ }^{18} \mathrm{O}=479 \pm 29$. The significantly lower, non-terrestrial ratio obtained by Avres, Plymate \& Keller (2006) is likely the result of their use of 1D model atmospheres. Several lines due to ${ }^{12} \mathrm{C}^{17} \mathrm{O}$ are present in the solar infrared spectrum but due to their extreme weakness we do not believe a trustworthy ${ }^{16} \mathrm{O} /{ }^{17} \mathrm{O}$ ratio can be inferred from them at this stage.

\subsection{Intermediate Mass Elements}

Sodium: Asplund et al. (2009b) based their 3D solar Na abundance determination on five weak $\mathrm{Na}$ I lines, which all yielded consistent results $(\sigma=0.03 \mathrm{dex})$. The predicted departures from LTE in $1 \mathrm{D}$ are slightly negative $(\approx-0.04$ dex $)$ and agree well between different studies (Allende Prieto. Asplund \& Fabiani Bendicho 2004, Shi. Gehren \& Zhao 2004, Takeda et al. 2003). The Na abundance we adopt here is slightly larger than the preliminary value presented in Asplund. Grevesse \& Sal $(2005)$.

Magnesium: The abundance of $\mathrm{Mg}$ can be inferred from two ionization stages, each with a multitude of seemingly clean, relatively weak lines (Lambert \& Luck 1978). Unfortunately, the $g f$-values for Mg I and Mg II are notoriously uncertain, as reflected in fairly large line-to-line abundance variations for both species. 
Departures from LTE in 1D line formation by $\mathrm{Mg}$ I have been considered by Zhao, Butler \& Gehren (1998) and T. Gehren (2008, private communication); the abundance corrections are small and positive. Abia \& Mashonkina (2004) and L. Mashonkina (2008, private communication) have made corresponding calculations for Mg II. With the predicted non-LTE effects, the mean Mg II abundance is brought into better agreement with the meteoritic abundance while $\mathrm{MgI}$ is further offset. Since we do not have greater confidence in either set of transition probabilities and non-LTE calculations are available for both species, we simply take a straight mean of all lines (Asplund et al. 2009b). The attached uncertainty is disappointingly large for such an important element.

Aluminium: Our recommended $\mathrm{Al}$ abundance comes from Asplund et al. (2009b), who performed an analysis of seven good, weak Al I lines with relatively well-determined $g f$-values. The departures from LTE are predicted to be small. We have adopted the non-LTE results from 1D calculations by Gehren et al. (2004), with the expectation that they will not be very different in 3D.

Silicon: Silicon is a critical element since it provides the absolute normalization for the meteoritic abundances (Sect. 4.1). Asplund (2000) carried out an early 3D-based LTE analysis of Si I and Si II lines and found $\log \epsilon_{\mathrm{Si}}=7.51 \pm 0.04$. With an improved 3D solar model and non-LTE corrections $(\approx-0.02$ dex $)$ taken from Shi et al. (2008), Asplund et al. (2009b) found the same value in their reanalysis, which we adopt here. Had we instead opted to use the Holweger \& Müller (1974) 1D semi-empirical model the result would have been a mere 0.02 dex higher.

Phosphorus: Caffau et al. (2007b) carried out a solar P determination using a CO5BOLD 3D solar model and found $\log \epsilon_{\mathrm{P}}=5.46(\sigma=0.04)$ from four P I lines. A slightly lower 3D-based value was obtained by Asplund et al. (2009b), who also included one more line: $\log \epsilon_{\mathrm{P}}=5.41 \pm 0.03(\sigma=0.03)$. We adopt this latter value on account of its smaller line-to-line scatter. No non-LTE investigation has been performed to date for these high-excitation P I lines. Departures from LTE are not expected to be significant however, a prediction supported by the small non-LTE effects for the similar S I lines (Takeda et al. 2005).

Sulphur: In their 3D-based analysis, Caffau et al. (2007a) obtained a disturbingly large line-to-line scatter: $\log \epsilon_{\mathrm{S}}=7.21 \pm 0.11(\sigma)$; this result includes the non-LTE corrections of Takeda et al. (2005). Very recently, the issue of the solar S abundance was revisited by Asplund et al. (2009b), who included a few additional lines. Their non-LTE abundance is slightly smaller and, importantly, shows a much reduced uncertainty: $\log \epsilon_{\mathrm{S}}=7.12 \pm 0.03(\sigma=0.03)$. The differences between Asplund et al. (2009b) and Caffau et al. (2007a) can be partly traced to the adopted equivalent widths, but this cannot be the whole explanation. Interestingly, Asplund et al. (2009b) concluded that the forbidden [S I] $1082 \mathrm{~nm}$ line returns too high an abundance by $0.3 \mathrm{dex}$, which may signal an unidentified blend, non-LTE effects or that the theoretical transition probability is in error. Caffau \& Ludwig (2007) on the other hand found excellent agreement with the meteoritic value for this line. They adopted however an outdated $g f$-value and a much smaller equivalent width, which we are unable to reconcile with the observed solar spectrum.

Potassium: Asplund et al. (2009b) based their 3D analysis on six K I lines, including the strong resonance line at $769.9 \mathrm{~nm}$. As in previous studies, the lineto-line scatter is disappointingly large for these lines, even when taking into account the theoretical non-LTE effects computed by Zhang et al. (2006). In terms 
of abundances, the non-LTE corrections are substantial for the two strongest lines $(\geq-0.2$ dex $)$.

Calcium: The solar Ca abundance can be determined from both Ca I and Ca II (Lambert \& Luck 1978), although there are problems with both species: most of the Ca I lines are rather strong, whilst the uncertainties in the transition probabilities for the Ca II lines are still unsatisfactory large (Opacity Project data assuming LS-coupling). Asplund et al. (2009b) found good agreement between the two ionization stages and obtained a final $\mathrm{Ca}$ abundance of $\log \epsilon_{\mathrm{Ca}}=6.34 \pm 0.04$ $(\sigma=0.04)$ from all Ca I and Ca II lines, including 1D non-LTE abundance corrections computed by Mashonkina. Korn \& Przybilla (2007). We note that the forbidden [Ca II] line at $732.3 \mathrm{~nm}$ implies an abundance which is fully consistent with the other transitions.

\subsection{Iron-peak Elements}

Scandium: With its relatively low ionization potential, Sc is prone to overionization via non-LTE effects (Zhang. Gehren \& Zhao 2008). The impact on abundances from Sc I lines is dramatic $(+0.15$ dex relative to LTE on average for the lines considered by Scott et al. 2009b), but predictably mild for Sc II $(-0.01 \mathrm{dex})$. Good lines, oscillator strengths and hyperfine constants are available for both ionization stages. With five weak lines of Sc I, twelve of Sc II and the non-LTE corrections of Zhang. Gehren \& Zhao (2008), Scott et al. (2009b) have produced 3D-based abundances showing very good agreement between the two ionization stages. In Table 1 we give the mean of the abundances from all lines of both species, where Sc II ultimately dominates because of the greater number of lines. The +0.10 dex difference between the new Sc abundance and the meteoritic one is intriguing.

Titanium: Very many good lines of Ti I are present in the Sun, virtually all with excellent atomic data. Whilst fewer lines of Ti II are available and the atomic data are not quite as good as for TiI, the situation is still relatively acceptable. The one glaring absence is a study of non-LTE effects for this element. As is the case for its neighbours $\mathrm{Sc}$ and $\mathrm{V}$, the low ionization potential of $\mathrm{Ti}$ and the resulting minority status of $\mathrm{Ti}$ I are expected to significantly skew the abundances it returns under the assumption of LTE. Using the improved 3D model atmosphere, Scott et al. (2009b) found stark disagreement between LTE abundances from Ti I and Ti II (Ti I: $\log \epsilon_{\mathrm{Ti}}=4.85 \pm 0.06$, Ti II: $\log \epsilon_{\mathrm{Ti}}=4.99 \pm$ 0.04 ), though with very low scatter from each species individually $(\sigma=0.03$ for both stages). In the absence of any non-LTE study, we favour Ti II in Table 1 by taking the mean of the two results weighted according to their total uncertainties; we only retain any weighting for the Ti I result because of the sheer number of excellent lines and oscillator strengths it includes.

Vanadium: Neutral vanadium has scores of excellent lines in the visible solar spectrum, whilst very few are available from V II, all extremely perturbed. On the other hand, VI is expected to show similarly strong non-LTE effects as verified for Sc I and suspected for Ti I, owing to the elements' similar potential for overionization. The impact of non-LTE line formation on $\mathrm{V}$ has not yet been the subject of a dedicated study. Scott et al. (2009b) obtain a 3D LTE abundance of $\log \epsilon_{\mathrm{V}}=3.83$ from V I lines. Scott et al.'s analysis of V II lines yields an abundance quite consistent with this corrected value, but the poor quality of the lines employed makes the result inconclusive. In the absence of any good results 
for V II, we adopt the V I value but adjust it by the mean non-LTE effect seen in Sc I and Cr I (+0.10 dex).

Chromium: The solar spectrum exhibits a large number of clean, weak lines of both neutral and once-ionised chromium. Unfortunately, no reliable oscillator strengths are available for CrII. Neither is any estimate of the impact of non-LTE effects on $\mathrm{Cr}$ available; given its relatively low ionization potential, $\mathrm{Cr}$ should consist mostly of $\mathrm{Cr}$ II in the Sun, possibly leading to non-LTE effects for Cr I. Recent non-LTE calculations by M. Bergemann (private communication) confirm departures from LTE at the level of +0.04 dex. Using their own measured oscillator strengths for Cr I, Sobeck, Lawler \& Sneden (2007) recently revised the solar chromium abundance. This result was updated by Scott et al. (2009b) using a 3D model. Given the much greater number of Cr I lines in the sample, and the poor quality of the Cr II oscillator strengths, we allow Cr I to dominate the value in Table 1 by taking the mean of abundances from all $\mathrm{Cr}$ I and $\mathrm{Cr}$ II lines together. The only reason we allow abundances from Cr II lines to enter into the mean is our suspicion of an as yet unquantified non-LTE effect for Cr I.

Manganese: Following years of confusion now traceable mostly to poor oscillator strengths, the solar manganese abundance was recently rederived by Blackwell-Whitehead \& Bergemann (2007), using their own improved Mn I g.fvalues, extensive hyperfine splitting data and the non-LTE calculations of Bergemann \& Gehren (2007). This result was updated by Scott et al. (2009b) using a 3D solar model, bringing the photospheric Mn abundance even closer to the meteoritic value. Mn I shows moderate non-LTE effects (about $+0.06 \mathrm{dex}$ ), consistent with its ionization potential being intermediate between the lower values seen in the lighter members of the iron group $\mathrm{Sc}, \mathrm{Ti}, \mathrm{V}$ and $\mathrm{Cr}$ and the higher values exhibited by the heavier elements $\mathrm{Fe}$, Co and Ni.

Iron: In many circumstances iron serves as a proxy for the overall metal content and as a reference against which other elemental abundances are measured. An intense debate long raged as to whether the solar Fe abundance inferred from Fe I lines should be $\log \epsilon_{\mathrm{Fe}} \approx 7.7$ (e.g. Blackwell. Lynas-Gray \& Smith 1995) or $\log \epsilon_{\mathrm{Fe}} \approx 7.5$ (e.g. Holweger, Kock \& Bard 1995), with the choice of equivalent widths, $g f$-values, microturbulence parameters and pressure-damping constants all playing a role. The advent of improved transition probabilities for Fe II lines (e.g. Hannaford et al. 1992, Schnabel. Kock \& Holweger 1999) and a proper treatment of pressure broadening (e.g. Anstee. O'Mara \& Ross 1997, Barklem. Piskunov \& O'Mara 2000a) strengthened the case for a low Fe abundance. An unsatisfactory combination of large abundance scatter, the presence of a free parameter (microturbulence), and a trend with excitation potential still remained with the Holweger \& Müller (1974) model, however (Grevesse \& Sauval 1999). Using a 3D hydrodynamical solar model and LTE line profile fitting, Asplund et al. (2000c) succeeded in achieving consistent results for Fe I and Fe II lines without invoking any microturbulence: $\log \epsilon_{\mathrm{Fe}}=7.45 \pm 0.05$.

The solar Fe abundance we advocate here comes from Scott et al. (2009b). The Fe I-based abundance of $\log \epsilon_{\mathrm{Fe}} \approx 7.52 \pm 0.05$ from weak lines is slightly higher than in Asplund et al. (2000c) on account of a slightly shallower mean temperature structure in the new 3D model and allowance of departures from LTE. The non-LTE abundance corrections have been estimated to be $+0.03 \mathrm{dex}$ for Fe I (Collet, Asplund \& Thévenin 2005; Korn, Shi \& Gehren 2003), although this depends on the still poorly-known inelastic H collisions (see Asplund 2005, and references therein). In fact, a more reliable abundance now comes from 
Fe II lines, thanks to the improved transition probabilities of Meléndez \& Barbuy (2009) and the broadening treatment of Barklem \& Aspelund-Johansson (2005). Together these imply $\log \epsilon_{\mathrm{Fe}} \approx 7.50 \pm 0.04(\sigma=0.04)$ with the same 3D model. We note that with the Holweger \& Müller (1974) model, there is a trend in Fe I abundances with excitation potential and a discrepancy of 0.14 dex between Fe I and Fe II. Both have disappeared with the 3D solar model (Fig. 6). Reassuringly, the situation regarding Fe has greatly improved over the past decade.

Cobalt: The fairly high ionization potential of $\mathrm{Co}$ and the availability of many good Co I lines, $g f$-values and hyperfine constants should make its solar analysis quite straightforward. Scott et al. (2009b) found a 3D LTE abundance of $\log \epsilon_{\mathrm{Co}}=4.88$, in agreement with the meteoritic value. Taking into account the surprisingly large non-LTE corrections of Bergemann (2008) changes the final abundance to $\log \epsilon_{\mathrm{Co}}=4.99 \pm 0.07(\sigma=0.05)$.

Nickel: The solar Ni abundance was estimated by Scott et al. (2009a) from 17 weak Ni I lines, showing a very small abundance scatter. The value given in Table

1 is a slight revision of this result (Scott et al. 2009b) based on the improved 3D model atmosphere that has been employed throughout this review.

\subsection{Neutron Capture Elements}

Quite some work on the solar abundances of the heavy elements has been carried out over the past decade, largely driven by improvements in atomic transition probabilities. In this context, we would like to particularly highlight the very careful laboratory measurements performed by the Wisconsin group (e.g. Lawler et al. 2009). In a beautiful series of papers, they have systematically and methodically measured radiative lifetimes, branching fractions, oscillator strengths, isotopic shifts and hyperfine constants for all the rare Earth elements: La (Lawler, Bonvallet \& Sneden 2001), Ce (Lawler et al. 2009), Pr (Sneden et al. 2009), Nd (Den Hartog et al. 2003), Sm (Lawler et al. 2006), Eu (Lawler et al. 2001b), Gd (Den Hartog et al. 2006), Tb (Lawler et al. 2001a), Dy (Sneden et al. 2009), Ho (Lawler. Sneden \& Cowan 2004), Er (Lawler et al. 2008), Tm, Yb and $\mathrm{Lu}$ (Sneden et al. 2009); the group has also carried out a similar study for Hf (Lawler et al. 2007). In their solar abundance analyses, they have employed the 1D Holweger \& Müller (1974) model atmosphere and assumed LTE for the spectrum synthesis. Although all the utilized transitions are from the majority ionization stage (once ionized) for the rare Earths, one still expects a minor temperature and thus model-atmosphere dependence, since the lines originate from atomic levels with low excitation potentials $\left(\chi_{\mathrm{exc}} \leq 1.5 \mathrm{eV}\right)$. The recommended solar abundances provided in Table 1 for these elements have been modified from the original sources by calculating the abundance difference between a 3D hydrodynamical model atmosphere and the Holweger \& Müller (1974) model, each computed using the same line formation code with consistent input data (Grevesse et al. 2009). For the majority of these lines, such (3D-HM) abundance corrections amount to $\approx-0.04$ dex. In addition, the $1 \mathrm{D}$ non-LTE effects on Eu II predicted by Mashonkina \& Gehren (2000) have been taken into account in the 3D result; non-LTE studies are not available for any other rare Earth elements.

A similar procedure has been implemented for Cd (Youssef. Doenszelmann \& Grevesse 1990), W (Holweger \& Werner 1982), Os (Quinet et al. 2006), Ir (Youssef \& Khalil 1988) and $\mathrm{Au}$ (Youssef 1986), all of which have only heavily blended transitions available in the solar spectrum, necessitating careful and time-consuming spec- 
trum syntheses. Due to blends, we consider the two As I lines employed by Gopka et al. (2001) too unreliable to base a meaningful abundance determination on and consequently do not provide a solar photospheric As abundance here. Similarly, we are unable to defend an estimate of the Sb abundance from the very weak and heavily perturbed, purported $\mathrm{Sb}$ I line at $323.2 \mathrm{~nm}$ reported by Ross \& Aller (1976).

For all other neutron capture elements, the adopted abundances come from a complete 3D-based reanalysis using the best atomic data and newly measured equivalent widths or spectrum synthesis for the most reliable lines. Grevesse et al. (2009) present the solar analyses of $\mathrm{Cu}, \mathrm{Zn}, \mathrm{Ga}, \mathrm{Ge}, \mathrm{Rb}, \mathrm{Sr}, \mathrm{Y}, \mathrm{Zr}, \mathrm{Nb}, \mathrm{Mo}, \mathrm{Ru}$, $\mathrm{Rh}, \mathrm{Pd}, \mathrm{Ag}, \mathrm{Cd}, \mathrm{Sn}, \mathrm{Ba}, \mathrm{Pb}$ and $\mathrm{Th}$, which we adopt throughout; the case of $\mathrm{Ru}$ is also discussed in Fivet et al. (2009). The available 1D non-LTE abundance corrections for Zn I (Takeda et al. 2005), Sr II (Mashonkina \& Gehren 2001) and Ba II (Mashonkina \& Gehren 2000) have been included in these estimates. For comparison purposes we have also repeated the line calculations with an assortment of 1D model atmospheres. We have found very good agreement $(<0.02 \mathrm{dex})$ with recent studies using the Holweger \& Müller (1974) model when allowance is made for differences in adopted transition probabilities and equivalent widths for Ge (Biémont et al. 1999), Sr (Barklem \& O’Mara 2000), Pd (Xu et al. 2006) and $\mathrm{Pb}$ (Biémont et al. 2000).

A handful of elements have previously been exposed to a 3D-based solar abundance analysis. Liung et al. (2006) made use of new transition probabilities for Zr II and employed the 3D solar model of Asplund et al. (2000b). Not surprisingly, the agreement with Grevesse et al. (2009) is very good. Eu has been the focus of Mucciarelli et al. (2008), who employed a 3D CO5BOLD hydrodynamical model and found a mean Eu abundance in perfect agreement with ours. The 3D LTE abundance for Hf from Caffau et al. (2008b) is only 0.02 dex higher than the corresponding value by Grevesse et al. (2009), who corrected the spectrum synthesis results of Lawler et al. (2007) for 3D effects rather than attempting to measure the equivalent widths of these weak and blended Hf II lines. Caffau et al. $(2008 \mathrm{~b})$ also performed a detailed investigation using 3D line profile fitting of the Th II $401.9 \mathrm{~nm}$ line, which is distorted by two Co I and V I lines (not V II as stated in their paper), as well as being in the red wing of a relatively strong feature from $\mathrm{Fe}$ I and $\mathrm{Ni}$. Interestingly, they argued that it is important to take into account the line asymmetry of the Fe I+Ni I blend introduced by the convective motions (e.g. Asplund et al. 2000b), which otherwise would lead to an overestimation of the Th abundance by $\approx 0.1$ dex. Unfortunately Caffau et al. (2008b) did not specify what abundances they used for the blending lines, so it is difficult to exactly reproduce their result; the value obtained by Grevesse et al. (2009) is consistent with that of Caffau et al., though smaller by 0.06 dex.

\subsection{Abundances from Sunspots: F, Cl, In, Tl}

The photospheric abundances of a few elements are not possible to measure using spectroscopy of the quiet Sun. The solar fluorine and chlorine abundances have instead been inferred from the infrared spectrum of sunspots using lines of $\mathrm{HF}$ (Hall \& Noves 1969) and $\mathrm{HCl}$ (Hall \& Noves 1972). Given the improvements in molecular data, observational quality and sunspot modelling in the intervening years since the original workds, it seems like a critical re-evaluation of these studies is warranted but not attempted here. The same holds for $\mathrm{Tl}$ 
(Lambert, Mallia \& Smith 1972). We note that in the meantime a more reliable estimate of the solar $\mathrm{Cl}$ may be provided by the abundance of nearby $\mathrm{H}$ II regions, which is $\log \epsilon_{\mathrm{Cl}}=5.32 \pm 0.07$ (García-Rojas \& Esteban 2007). Recently, Vitas et al. (2008) have revisited the solar In abundance. In contrast to previous studies based on the quiet Sun, they find an abundance from a sunspot spectrum in agreement with the meteoritic value.

\subsection{Indirect Determinations: Noble Gases}

Due to their high excitation potentials, there are no photospheric lines of the noble gases helium, neon, argon, krypton and xenon available in the solar spectrum, which forces one to estimate the solar abundances of these elements through indirect methods.

Helium: A highly accurate He abundance determination is possible through helioseismology. The technique utilizes the change in the adiabatic index $\Gamma_{1}$ in the He II ionization zone that takes place at a solar radius of $r / R_{\odot} \approx 0.98$. The result is quite insensitive to the reference solar model employed for the inversion, but does depend on the adopted equation-of-state. Basu \& Antia (2004) found a He mass fraction in the convection zone of $Y_{\mathrm{s}}=0.2485 \pm 0.0034$, which corresponds to a He abundance by number of $\log \epsilon_{\mathrm{He}}=10.93 \pm 0.01$. The uncertainty essentially reflects the difference between the MHD (Mihalas, Däppen \& Hummer 1988) and OPAL (Rogers \& Nayfonov 2002) equation-of-state, but there is also a minor dependence on the assumed metallicity of the reference solar model for the inversion (Trampedach. Däppen \& Baturin 2006). A similar value is obtained when calibrating solar interior models to achieve the correct mass, luminosity and radius at the present solar age, if the chemical composition of Grevesse \& Sauval $(1998)$ is adopted and diffusion is considered. With the solar abundances of Asplund, Grevesse \& Sauval (2005) on the other hand, the calibrated proto-solar He abundance would imply a surface value lower than the helioseismic measurement by 0.02 dex. Such models are also in conflict with other helioseismological evidence such as the depth of the convection zone and the sound speed variation with depth, as discussed in Sect. 4.3.

Neon: The Ne abundance can be inferred from X-ray and UV spectroscopy of the solar corona and solar flares as well as directly from the solar wind. The interpretation is, however, complicated by the so-called first ionization potential (FIP) effect: elements with ionization potential $\chi_{\text {ion }}<10 \mathrm{eV}$ are enhanced in the upper solar atmosphere and solar wind compared with their photospheric values, whilst the high ionization elements are not or only slightly affected (Feldman \& Widing 2003). Rather than measuring the Ne abundance directly, a common approach is to determine the abundance relative to a reference element and assume that the ratio is the same in the photosphere. Here $\mathrm{O}$ is the reference element of choice, although we note that there is still a significant difference in ionization potential between $\mathrm{O}(13.6 \mathrm{eV})$ and $\mathrm{Ne}(21.6 \mathrm{eV})$, which could potentially introduce a systematic error given our poor understanding of the physical reasons for the FIP effect. The degree of chemical separation varies significantly, being more severe in regions of higher solar activity. Young (2005b) found a Ne/O ratio of $0.175 \pm 0.031$ for the quiet Sun, which is the value we adopt here to convert our photospheric $\mathrm{O}$ abundance to a Ne content. This result is particularly appealing for our purposes since Young (2005a) has demonstrated that the average quiet Sun does not show any FIP effect, a conclusion which is further corrob- 
orated when using the solar abundances given herein. This ratio is consistent with the results of Schmelz et al. (2005) for active regions $(0.175 \pm 0.074)$ and for the solar wind $(0.14 \pm 0.03)$ by Bochsler (2007). The corresponding ratio in solar energetic particles is very similar and remarkably constant: $0.152 \pm 0.006$ (Reames 1999), which was adopted by Asplund, Grevesse \& Sauval (2005). In spite of its very small uncertainty, here we weight this result less than from the quiet Sun due to the potential modification through the FIP effect. Together with our recommended $\mathrm{O}$ abundance, the photospheric Ne abundance becomes $\log \epsilon_{\mathrm{Ne}}=7.93 \pm 0.09$.

Our value is smaller than the solar flare determination $\left(\log \epsilon_{\mathrm{Ne}}=8.11 \pm 0.12\right)$ by Landi. Feldman \& Doschek (2007), but we consider the quiet Sun a more reliable indicator than solar flares due to possible fractionation processes in the latter. While the Ne abundance $\left(\log \epsilon_{\mathrm{Ne}}=7.96 \pm 0.13\right)$ of Bochsler (2007) agrees with ours, there are significant additional uncertainties in this result on account of using $\mathrm{He}$ as a reference element, as it is known to be highly variable in the solar wind.

Drake \& Testa (2005) have argued on the basis of X-ray spectroscopy of nearby stars that the $\mathrm{Ne} / \mathrm{O}$ ratio is significantly higher (0.41) than the solar ratios discussed above. Their sample is, however, heavily biased towards active stars, which experience an inverse FIP effect: elements with $\chi_{\text {ion }}>10 \mathrm{eV}$ are enhanced in the corona compared with low ionization potential species (Güdel 2004). Indeed, Robrade. Schmitt \& Favata (2008) show that there is a systematic trend in $\mathrm{Ne} / \mathrm{O}$ with activity level such that other inactive stars have similar low ratios to the Sun. One would therefore suspect that the high coronal Ne/O ratio inferred by Drake \& Testa (2005) is a consequence of the difference in ionization potential, making Ne more susceptible to fractionation than $\mathrm{O}$ in high activity environments. As will be further discussed in Sect. 4.2, a more reliable stellar gauge of the solar Ne abundance is provided by OB stars in the solar neighborhood. Both the Ne/O ratio $(0.20 \pm 0.03)$ and the absolute Ne abundance $\left(\log \epsilon_{\mathrm{Ne}}=8.08 \pm 0.03\right)$ estimated in this way are in very good agreement with the solar values we adopt here when the effects of solar diffusion and Galactic chemical enrichment over the past 4.5 Gyr are accounted for (Przybilla. Nieva \& Butler 2008).

Argon: As discussed in detail by Lodders (2008), there are a wide range of methods for inferring the solar Ar abundance: solar wind measurements, solar flares and energetic particles, nuclear statistical equilibrium with the abundances of the nearby $\alpha$-elements ${ }^{28} \mathrm{~S}$ and ${ }^{40} \mathrm{Ca}$, and comparison with other solar system objects (Jupiter) and the solar neighborhood (B stars, planetary nebulae, H II regions). We follow the procedure of Lodders (2008) but employ our preferred values for the photospheric abundances of $\mathrm{O}$ (for the solar wind, flares and energetic particles), Kr and Xe (for analyses of Jupiter), and the lower He abundance appropriate for the upper atmosphere instead of the photospheric value (for solar wind analyses). The quoted errors for all methods in the literature are only the dispersions of the measurements, so likely underestimate of the true uncertainties. We therefore take a straight unweighted mean of all techniques, which leads to $\log \epsilon_{\mathrm{Ar}}=6.40 \pm 0.13$. Our value is $0.1 \mathrm{dex}$ lower than the result of Lodders (2008) but 0.2 dex higher than Asplund, Grevesse \& Sauval (2005), which was based only on the Ar/O ratio in solar energetic particles (Reames 1999).

Krypton: The Kr abundance has been estimated from interpolation of the theoretical $s$-process production rates, since the cross-sections for neutron capture 
of nearby pure $s$-isotopes have been accurately measured experimentally (Palme $\&$ Beer 1993). Our value accounts for a slight revision of the photospheric Si abundance, which serves as a normalization of the $s$-process predictions. This $\mathrm{Kr}$ estimate agrees well with the value inferred from the solar wind $\mathrm{Kr} / \mathrm{O}$ ratio together with our photospheric O abundance (Geiss, Gloeckler \& von Steiger 1994; Heber et al. 2008b).

Xenon: The procedure to estimate the Xe content is the same as for $\mathrm{Kr}$ (Käppeler 2005). For Xe, however, this $s$-process estimated value is 0.4 dex lower than deduced from the solar wind as measured from lunar regoliths (Geiss. Gloeckler \& von 1994) and the Genesis space probe (Heber et al. 2008b). This may suggest that Xe has experienced fractionation through the FIP effect in spite of its relatively high ionization energy $\left(\chi_{\text {ion }}=12.1 \mathrm{eV}\right)$.

\subsection{Solar Isotopic Abundances}

As a convenience to the reader, in Table 3 we give our best estimates of the protosolar isotopic fractions for each element. In most cases the terrestrial isotopic abundance ratios as recommended by International Union of Pure and Applied Chemistry (Rosman \& Taylor 1998) have been adopted as representative of the solar system values; direct solar isotopic information can only be gleaned from CO vibration-rotation lines in the infrared spectrum of the Sun (e.g. Scott et al. 2006), although with comparatively large error bars by terrestrial standards. It should be noted that except for $\mathrm{C}$ and $\mathrm{O}$, the isotopic fractions given here are the more conservative "representative isotopic composition" from the compilation of Rosman \& Tavlor (1998), in contrast to Lodders (2003) who opted for the "best measurement from a single terrestrial source" from the same reference. For most volatile elements more reliable isotopic fractions are available from alternative solar system sources due to strong depletion of terrestrial samples, as discussed below.

The proto-solar deuterium abundance can be estimated from observations of Jupiter. Using ISO spectra of the Jovian atmosphere and taking into account an expected 5-10\% enrichment during the planet's formation, Lellouch et al. (2001) obtained a proto-solar abundance of $\mathrm{D} / \mathrm{H}=(2.1 \pm 0.4) \cdot 10^{-5}$. An alternative approach makes use of the measured enrichment of the ${ }^{3} \mathrm{He} /{ }^{4} \mathrm{He}$ ratio in the solar wind in comparison with the proto-solar ratio ${ }^{3} \mathrm{He} /{ }^{4} \mathrm{He}=(1.66 \pm 0.05)$. $10^{-4}$ inferred from Jupiter (Mahaffy et al. 1998), since the additional ${ }^{3} \mathrm{He}$ can be attributed to D-burning in the Sun (Gloeckler \& Geiss 2000). Together with the solar wind ratio ${ }^{3} \mathrm{He} /{ }^{4} \mathrm{He}=(4.53 \pm 0.03) \cdot 10^{-4}$ from Genesis measurements (Heber et al. 2008a), the Jovian ratio implies $\mathrm{D} / \mathrm{H}=(1.96 \pm 0.3) \cdot 10^{-5}$; most of the uncertainty comes from the poorly-known He fractionation in the solar wind. From the two methods, we finally estimate a proto-solar abundance of $\mathrm{D} / \mathrm{H}=(2.0 \pm 0.2) \cdot 10^{-5}$. This is consistent with the present-day local interstellar medium abundance (Linsky et al. 2006), which implies only a small degree of astration over the past $4.5 \mathrm{Gyr}$.

The ${ }^{12} \mathrm{C} /{ }^{13} \mathrm{C}$ ratio does not appear to vary significantly between different solar system sources, so we simply adopt the accurate terrestrial reference value of $\mathrm{Vi}$ enna Peeddee belemnite: ${ }^{12} \mathrm{C} /{ }^{13} \mathrm{C}=89.4 \pm 0.2$ (Coplen et al. 2002). We note that the "representative isotopic composition" from Rosman \& Taylor (1998) corresponds to the significantly more uncertain value ${ }^{12} \mathrm{C} /{ }^{13} \mathrm{C}=92 \pm 7$. The ratio inferred from the solar photosphere using CO lines $(86.8 \pm 3.8)$ is consistent with 
the terrestrial value (Scott et al. 2006).

The ${ }^{14} \mathrm{~N} /{ }^{15} \mathrm{~N}$ ratio varies tremendously between various solar system objects, from 100 to 450 (Owen et al. 2001). Here we adopt the Jovian value $435 \pm 57$ as representative of the proto-solar ratio, in agreement with values in the interstellar medium and carbonaceous chondrites (Meibom et al. 2007).

Oxygen isotopic abundances display a variability of up to a few percent within the solar system. The best values to adopt for the proto-solar oxygen isotopic ratios are still debated (e.g. Hashizume \& Chaussidon 2005, Ireland et al. 2006). The first results from the Genesis mission should appear very soon (McKeegan et al. 2008), which will hopefully shed some light on the issue. Until such further clarification, we adopt the terrestrial values represented in Vienna Standard Mean Ocean Water: ${ }^{16} \mathrm{O} /{ }^{18} \mathrm{O}=498.7 \pm 0.1$ and ${ }^{16} \mathrm{O} /{ }^{17} \mathrm{O}=2632 \pm 7$ (Coplen et al. 2002). The solar photospheric ratio ${ }^{16} \mathrm{O} /{ }^{18} \mathrm{O}=479 \pm 29$ obtained by Scott et al. (2006) is consistent with the terrestrial value, but with larger uncertainties (although in fairness the terrestrial "representative isotopic composition" from Rosman \& Tavlor (1998) is equally uncertain: $487 \pm 35$ ). Surprisingly, Ayres, Plymate \& Keller (2006) found strongly sub-terrestrial ratios (e.g. ${ }^{16} \mathrm{O} /{ }^{18} \mathrm{O}=440 \pm 20$ ) from their analysis of the solar $\mathrm{CO}$ lines, probably due to an inadequate consideration of the differing temperature sensitivities of the isotopomer transitions with 1D model atmospheres (Scott et al. 2006). Lines from ${ }^{12} \mathrm{C}^{17} \mathrm{O}$ are also visible in the solar infrared spectrum, but due to their extreme weakness their reliability as abundance indicators is questionable.

For Ne, Ar, Kr and Xe we adopt the recommended values of Wieler (2002) derived from solar wind and lunar sample analyses. Very similar ratios have recently been presented from preliminary studies of solar wind samples from the Genesis mission (Heber et al. 2008b). For Mo, Dy, Er, Yb and Lu we have exchanged the Rosman \& Tavlor (1998) values with the more recent measurements given in Lodders, Palme \& Gail (2009). Similarly the proto-solar values given here for the radioactive nuclides have been taken from the same source.

\subsection{Conversion From Photospheric to Bulk Abundances}

The elemental abundances given in Table 1 are those currently present in the solar photosphere and by extension the whole convection zone. Due to the combined effects of thermal diffusion, gravitational settling and radiative acceleration (often referred to collectively as diffusion) over the past $4.56 \mathrm{Gyr}$, the values in Table 1 differ slightly from the proto-solar chemical composition. In comparison with present-day photospheric values, the bulk composition of the Sun is higher by 0.05 dex for helium and 0.04 dex for the heavier elements (Turcotte \& Wimmer-Schweingruber 2002). The uncertainty in the overall diffusion correction is judged to be about 0.01 dex. The element-to-element variation in the expected diffusion is very small $(<0.01 \mathrm{dex})$ and therefore currently not observationally testable by a comparison of photospheric and meteoritic abundances. We note that Lodders (2003) adopted a somewhat larger correction, $0.074 \mathrm{dex}$, than that of Turcotte \& Wimmer-Schweingruber (2002). She based her estimate on the results of Boothroyd \& Sackmann (2003), who computed their solar models using a less detailed diffusion model. 


\subsection{Solar metallicity}

With the solar chemical composition we recommend here, the mass fractions of $\mathrm{H}$, He and metals in the present-day photosphere become $X=0.7381, Y=0.2485$ and $Z=0.0134$. The respective values for the bulk composition are $0.7154,0.2703$ and 0.0142 . In other words, the solar metallicity is no longer the canonical $2 \%$ recommended by Anders \& Grevesse (1989) but rather a substantially smaller $1.4 \%$. Indeed, the intervening years have seen a steady decrease in $Z / X$ from 0.027 to our preferred value of 0.018. This is illustrated in Table 4 , which collects the resulting mass fractions from a number of widely used compilations of the solar chemical composition over the past two decades. Our metallicity reverses this declining trend of $Z$ with time by being slightly larger than advocated by Asplund. Grevesse \& Sauval (2005), which is mainly the result of the somewhat higher $\mathrm{C}, \mathrm{O}, \mathrm{Ne}$ and $\mathrm{Fe}$ abundances.

For completeness, we note that the He abundances recommended by Anders \& Grevesse (1989) and Grevesse \& Noels (1993) were based on calibration of solar models and hence corresponded to bulk composition; here we have substituted those with the helioseismic convection zone He content (Sect. 3.9) when computing the presentday photospheric values. Finally, from Table 4 it is clear that the surface $\mathrm{He}$ abundance employed by Lodders (2003) is too low on account of adopting a too efficient elemental diffusion, as discussed in Sect. 3.11.

\section{OTHER METHODS FOR INFERRING SOLAR ABUNDANCES}

\subsection{Meteorites}

Meteoritic data on the elemental abundances constitute an excellent comparator for the results from the solar photosphere presented above. Not all meteorites are suitable for the purpose though; only a very small subsample are believed to accurately reflect the chemical composition of the proto-solar nebula, since the majority have undergone varying degrees of fractionation and alteration. The most primitive, undifferentiated meteorites in this respect are the so-called CI (or C1) carbonaceous chondrites, of which there are only five known: the Alais, Ivuna, Orgueil, Revelstoke and Tonk meteorites (Lodders 2003). Of these, Orgueil is the by far largest and has therefore been exposed to the greatest number of analyses. It should be noted that while CI chondrites are believed not to have been noticeably chemically fractionated, they have still been severely modified mineralogically by aqueous alteration and thermal metamorphism. The main advantage of meteorites as a cosmic abundance standard is the extraordinarily high precision obtained, even for isotopic abundances, through mass spectroscopy. It comes at a cost, however, since the volatile elements $\mathrm{H}, \mathrm{C}, \mathrm{N}, \mathrm{O}$ and the noble gases - which also happen to be the most abundant elements - have been severely depleted. As a consequence, the abundances in meteorites are pegged to the Si content, rather than $\mathrm{H}$ as is the case in solar and stellar spectroscopy.

The mean abundances of CI chondrites presented in Table 1 have been appropriated from the data carefully compiled by Lodders. Palme \& Gail (2009). To place the meteoritic abundances (defined as $N_{\mathrm{Si}}=10^{6}$ ) on the same absolute scale as for the photosphere (defined as $\log \epsilon_{\mathrm{H}}=12.00$ ), we have renormalized their data such that the meteoritic and photospheric Si abundances agree. The relation between the two scales is thus specified as $\log \epsilon_{\mathrm{X}}=1.51+\log N_{\mathrm{X}}$. 
In principle the conversion factor could employ additional elements besides $\mathrm{Si}$; Anders \& Grevesse (1989) for example used Na, Mg, Si, Ca, V, Cr, Co, Ni, Y, $\mathrm{Zr}, \mathrm{Nb}$ and Mo, while Lodders, Palme \& Gail (2009) included as many as 39 elements. In practice, however, this does not change the result noticeably but it introduces some degree of arbitrariness in the selection of elements to include in this exercise. Fig. 7 shows the difference between the thus obtained photospheric and CI chondrite abundances, highlighting the excellent correspondence between the two methods. Naturally all volatile elements are depleted in the meteorites, whereas $\mathrm{Li}$ is depleted in the solar photosphere. For the other 45 elements with a purported photospheric uncertainty less than $25 \%$, the mean difference between photospheric and meteoritic abundances is $0.00 \pm 0.05$ dex. The photospheric and meteoritic abundances differ by more than the total combined uncertainty for only 10 out of the 57 non-volatile elements (expected $\approx 18 / 57$ ), which suggests that our estimated errors are in fact slightly too conservative. It should be emphasized that this excellent agreement cannot be interpreted as an absence of any significant diffusion in the Sun (Sect. 3.11), as the meteoritic scale is set by $\mathrm{Si}$; all elements heavier than $\mathrm{He}$ are fractionated by $\approx 0.04$ dex relative to $\mathrm{H}$ but hardly at all relative to each other.

In spite of the excellent agreement overall illustrated in Fig. 7, there are a few noticeable exceptions. Of the ten non-depleted elements where the solar and meteoritic abundances differ by more than 0.1 dex (25\%), this can in all cases be blamed on uncertain photospheric analyses. Indeed, in many cases the two methods still agree marginally within the combined errors ( $\mathrm{Cl}, \mathrm{Au}, \mathrm{Tl})$. We note a significant improvement for $\mathrm{W}$ compared with previous compilations of the solar chemical composition $(\Delta \log \epsilon=+0.49$ dex in Asplund, Grevesse \& Sauval (2005) while here the difference amount to $+0.20 \mathrm{dex})$; we suspect that unidentified blends and/or erroneous continuum placement may be at fault for the persisting discrepancy, as well as for $\mathrm{Rb}$ and $\mathrm{Hf}$. For Co we reckon that the non-LTE corrections by Bergemann (2008) are somewhat overestimated. The remaining three elements $\mathrm{Rh}, \mathrm{Ag}$ and $\mathrm{Pb}$ have had their photospheric values determined from transitions originating either in the ground state or from low-excitation levels of the neutral species (also true for $\mathrm{Pd}, \mathrm{W}$ and $\mathrm{Au}$ ), which are prone to non-LTE effects in their excitation and ionization populations (Asplund 2005).

\subsection{Solar Neighborhood}

Independent estimates of the chemical composition of the Sun are provided by solar-type stars, OB stars, HiI regions like the Orion nebula and the interstellar medium in the solar neighborhood. In order to make a meaningful comparison one must first account for the effects of diffusion in the Sun and Galactic chemical enrichment. The former amounts to 0.04 dex for metals (Turcotte \& Wimmer-Schweingruber 2002, cf. Sect. 3.11) while the latter is typically predicted to be $0.05-0.15$ dex depending on the element in question (Chiappini. Romano \& Matteucci 2003; Prantzos 2008).

Whether the Sun is somehow special in its chemical composition relative to other FG dwarfs in the Galactic thin disk has attracted attention over the years (e.g. Gustafsson 2008, Robles et al. 2008, and references therein). It should be borne in mind that most large-scale abundance analyses of Galactic thin disk stars to date (e.g. Bensby et al. 2005, Edvardsson et al. 1993, Fuhrmann 2008, Ramírez. Allende Prieto \& Lambert 2007, Reddy et al. 2003) have been 
performed differentially relative to the Sun to remove the uncertainties from $g f$-values and to minimize systematic errors due to inadequacies in the stellar modelling. These studies therefore reveal little about the absolute abundances of the stars but do imply that the Sun is a rather ordinary thin disk G dwarf for its age. In his volume-complete sample of stars within 25 pc, Fuhrmann (2008) found a mean spectroscopic metallicity of $[\mathrm{Fe} / \mathrm{H}]=-0.02 \pm 0.18$. Similarly, among the stars within $40 \mathrm{kpc}$ having an isochrone age of 4-6 Gyr, Holmberg, Nordström \& Andersen (2008) obtained a mean metallicity of $[\mathrm{Fe} / \mathrm{H}]=-0.00 \pm 0.10$. In their study of 11 solar twins, Meléndez et al. (2009) found that the solar X/Fe ratios did not differ by more than 0.06 dex from the mean of the solar twin sample for any of the 23 investigated elements.

In the past, the Sun appeared to be metal-rich compared with, for example, young OB stars in the solar neighborhood, despite the expected chemical enrichment over the past $4.5 \mathrm{Gyr}$. In the meantime, the solar abundances have decreased and the hot star abundances have increased. The latter have also become more accurate, as reflected in greatly diminished uncertainties (e.g. Daflon \& Cunha 2004, Morel 2008, Nieva \& Przvbilla 2006). Arguably the most accurate result to date is the analysis of Przybilla. Nieva \& Butler (2008), who have carried out a non-LTE study of six nearby B-type main sequence stars. Their results are presented in Table 5, for $\mathrm{S}$ we give the mean abundance from Morel et al. (2006) while the Ar abundance is taken from Lanz et al. (2008). When accounting for diffusion in the Sun, there is good agreement between the solar and hot star abundances for $\mathrm{O}, \mathrm{Ne}$ and $\mathrm{Mg}$ in particular. While the situation has greatly improved compared with the solar abundances of Grevesse \& Sauval (1998) and earlier compilations, there is still a tendency for the solar $\mathrm{C}, \mathrm{N}$ and $\mathrm{Fe}$ abundances to be somewhat higher than in the B stars, contrary to expectations. It is unclear whether the solution can be found in the solar or B star analyses or, if a real difference indeed exists, perhaps due to infall of low-metallicity gas to the solar neighborhood.

The abundances of nearby $\mathrm{H}$ II regions like the Orion nebula also provide constraints on the solar chemical composition. In Table 5 we provide the values from Esteban et al. (2004), Esteban et al. (2005) and García-Rojas \& Esteban (2007) interpolated to a Galacto-centric radius of $8 \mathrm{kpc}$. The observed scatter between individual $\mathrm{H}$ II regions is now quite small and the inferred present-day Galactic abundance gradient well determined. It is worth remembering that there are multiple potential pitfalls in the analyses of $\mathrm{H}$ II regions, including corrections for unseen ionization stages and the effects of temperature fluctuations in the nebula. Furthermore, abundances derived from recombination (permitted) lines are often significantly larger than the corresponding values from collisionally excited (forbidden) lines. Finally, dust condensation needs to be taken into account for some elements. For $\mathrm{C}$ and $\mathrm{O}$ this somewhat uncertain dust correction amounts to $\approx 0.1$ dex in Orion (Esteban et al. 2004). Volatile elements like Ne and S are not expected to form dust but refractory elements like $\mathrm{Mg}$ and Fe are predominantly in the solid phase, so it is therefore not possible to deduce a meaningful total elemental abundance for them. Overall, the agreement between the Sun and H II regions is very satisfactory when accounting for diffusion and a minor chemical enrichment over the past $4.5 \mathrm{Gyr}$.

Because of the generally lower temperatures encountered and the consequentially larger degree of dust condensation, the interstellar medium is a less reliable chemical composition gauge than H II regions (Jenkins 2004). Indeed, rather 
than setting constraints on the solar abundances, the Sun is often used to infer the amount in the solid phase in the interstellar medium (e.g. Sofia \& Meyer 2001). The local abundances of the volatile elements C (Sofia et al. 2004), N (Jensen, Rachford \& Snow 2007) and O (Cartledge et al. 2006, Jensen, Rachford \& Snow 2005) in the interstellar medium support our recommended solar values in general. With the older, high $\left(\log \epsilon_{\mathrm{O}}=8.83-8.93\right)$ solar abundances preferred by Grevesse \& Sauval (1998) and Anders \& Grevesse (1989) it becomes very challenging to hide sufficient amounts of $O$ in dust (Jensen 2007). The gas phase abundances of the refractory elements is only a small fraction of the total abundance in both the cold and warm interstellar medium, preventing them from setting any stringent constraints.

Also listed in Table 5 are predictions from models of Galactic chemical evolution (Chiappini, Romano \& Matteucci 2003). Indeed, with these values it becomes difficult to reconcile the solar abundances with the results from OB stars and $\mathrm{H}$ II regions for some elements like Fe. It is important to bear in mind though that the models have varying degrees of reliability for different elements. The evolutionary predictions for $\mathrm{O}, \mathrm{Ne}$ and $\mathrm{Mg}$ should be the most accurate, as they are produced in hydrostatic burning and ejected by SNeII; in fact for these elements there is good agreement between the Sun and its local neighborhood. All other elements are either produced in explosive burning and therefore dependent on the adopted mass-cut in the supernova explosion, or have a non-negligible contribution from SNe Ia or AGB stars, making theoretical estimates rather uncertain. One may conclude that the local Galactic volume has had a smaller star formation rate than assumed in these chemical evolution models. This inference is born out empirically by the observed age-metallicity relation, which Holmberg. Nordström \& Andersen (2008) estimated to be only $0.018 \mathrm{dex} / \mathrm{Gyr}$ for $[\mathrm{Fe} / \mathrm{H}]$ in the solar neighborhood, i.e. only half of the predictions given in Table 5. An alternative explanation would be recent Galactic infall of more pristine gas.

\subsection{Helioseismology}

The situation is decidedly less encouraging when attempting to assess the solar abundances by means of helioseismology. Since the various solar oscillation p-modes penetrate to different depths, it is possible to thereby map the variation of the sound speed in the solar interior, which can be compared with the predicted values from solar structure models (see Basu \& Antia 2008, for a detailed review). With the Grevesse \& Sauval (1998) abundances, the predicted sound speed as a function of depth from standard solar models shows a very good agreement with the helioseismic values. When instead employing the revised values of Asplund. Grevesse \& Sauval (2005), this is no longer the case (e.g. Bahcall et al. 2005, Basu \& Antia 2004, 2008, Delahave \& Pinsonneault 2006, Guzik. Watson \& Cox 2005, Turck-Chièze et al. 2004). In this context it is perhaps fair to say that 'better is worse'. The sound speed discrepancy most clearly shows up immediately below the bottom of the convection zone, as illustrated in Fig. 8. Also with the Grevesse \& Sauval (1998) abundances there was a disagreement in this region but it is greatly aggravated by the further lowering of the solar $\mathrm{O}$ and $\mathrm{Ne}$ abundances and to a lesser extent by the reassessment of $\mathrm{C}$, $\mathrm{N}$ and Fe. With the solar chemical composition recommended here, the situation is alleviated somewhat due to the slightly higher abundances of these elements 
and consequently larger opacity in the radiative interior but the deviation is still highly significant.

It is not only the sound speed variation that is at variance when using the new solar abundances. The inferred depth of the convection zone is now too shallow: $R_{\mathrm{BCZ}} \approx 0.725 \mathrm{R}_{\odot}$ instead of the helioseismic measurement $0.7133 \pm 0.0005 \mathrm{R}_{\odot}$ (Basu \& Antia 2004). Furthermore, the resulting He abundance when calibrating solar interior models to achieve the correct solar luminosity and temperature at the solar age is similarly inconsistent with the value inferred from helioseismology (see Sect. 3.9): $Y_{\mathrm{S}} \approx 0.238$ compared with the observed $0.2485 \pm 0.0034$ (Basu \& Antia 2004), where $Y_{\mathrm{S}}$ is the present-day He mass fraction at the surface. The problem also persists in the solar core both as implied by low-degree p-modes (Chaplin et al. 2007) and by gravity modes (García et al. 2007). Thus the problem exists both in the convective envelope, the radiative interior and in the core.

Following the initial revisions of the solar $\mathrm{C}$ and $\mathrm{O}$ abundances by Allende Prieto. Lambert (2001) and Allende Prieto, Lambert \& Asplund (2002), many possible solutions have been put forward to explain the problem albeit to date with little success. The most obvious explanation would be that the opacities are underestimated. Bahcall et al. (2005) estimated that a $10-20 \%$ increase of the OPAL opacities over a relatively substantial fraction of the solar interior $\left(R=0.4-0.7 \mathrm{R}_{\odot}\right.$ or equivalently $T=2-5 \cdot 10^{6} \mathrm{~K}$ ) would be required; Christensen-Dalsgaard et al. (2009) found even slightly more stringent requirements with the Asplund. Grevesse \& Sauva (2005) solar abundances. Serenelli et al. (2009) have investigated the differences with the solar chemical composition presented here and conclude that $\leq 12 \%$ extra opacity is required to restore the previous agreement in sound speed. Little suggests, however, that current atomic physics calculations for stellar interiors are missing such a substantial fraction of the opacity. For example, Badnell et al. (2005) showed that opacities from the Opacity Project are at most $3 \%$ larger than the corresponding OPAL values in the relevant temperature range. While unlikely to cure the problem by itself, it is perhaps wise to bear in mind that a similar situation for pulsating stars in fact existed in the 1980s, which found an unexpected resolution with the more comprehensive calculations by the OPAL and OP collaborations.

There are other ways to compensate for the lower opacities resulting from the lower $\mathrm{C}, \mathrm{N}, \mathrm{O}$ and $\mathrm{Ne}$ abundances. One is that the radiative interior contains more metals than expected from the photospheric abundances and standard models for diffusion. Asplund et al. (2004) first suggested that the diffusion efficiency may be seriously underestimated but the required elemental settlement is about twice the predicted value. Furthermore, while this may remedy the sound speed discrepancy, problems still remain in terms of the He abundance and depth of the convection zone (Guzik, Watson \& Cox 2005, Montalbán et al. 2004, Yang \& Bi 2007). A hypothetical late accretion of metal-depleted gas during the formation of the Sun runs into the same trouble (Castro, Vauclair \& Richard 2007; Guzik, Watson \& Cox 2005). Alternatively, the reduced O abundance could conceivably be compensated by a corresponding but larger increase in the Ne abundance (Antia \& Basu 2005). Bahcall, Basu \& Serenelli (2005) estimated that a factor of three (0.5 dex) higher Ne content compared with the recommended values by Asplund, Grevesse \& Sauval (2005), together with minor adjustments of $\mathrm{C}, \mathrm{N}, \mathrm{O}$ and Ar within their associated uncertainties would restore the good agreement found with the Grevesse \& Sauval (1998) solar composition. There 
was initial excitement when Drake \& Testa (2005) argued for exactly such an increase in $\mathrm{Ne}$ based on inferences from coronal spectroscopy of other stars but there is in fact little support for this idea, as explained in Sect. 3.9. The here recommended abundance of $\mathrm{Ne}$ as well as of $\mathrm{C}, \mathrm{N}, \mathrm{O}$ and $\mathrm{Fe}$ are $\approx 0.05 \mathrm{dex}$ higher than in Asplund, Grevesse \& Sauval (2005). This shift only partly allays the solar modelling discrepancy, as seen in Fig. 8.

Given that the problem exceeds the likely uncertainties in existing input data (e.g. opacities, diffusion, abundances), the only remaining option on the solar interior side seems to be to invoke some physical process not yet accounted for in standard solar models. It is perhaps suggestive that the largest deviation occurs immediately below the convection zone. Arnett. Meakin \& Young (2005) have proposed that internal gravity waves generated by the convective motion at the base of the convection zone propagate inwards and deposit their energy in the radiative zone. The ensuing structure changes are similar to an opacity enhancement, thus going in the right direction. In addition, the gravity waves induce additional mixing, which would also help. Young \& Arnett (2005) have investigated these processes using multi-dimensional hydrodynamical simulations of stellar convection zones and found qualitative improvements in many stellar regimes. Due to the computationally very challenging nature of the hydrodynamics, the modelling is unfortunately not yet able to quantitatively predict whether the effects are sufficient to explain the helioseismic signature.

Thus, most suggestions to resolve the solar modelling discrepancy have already been ruled out or are considered improbable. The few possibilities still standing do so largely because no detailed modelling has as yet been forthcoming to properly put the proposals to test. It is possible though that a combination of several factors could be the explanation, but such fine-tuning would seem rather contrived. Should no likely solution be found, it would suggest that the photospheric abundances presented herein are wrong. Given the discussion in Sect. 2 and Sect. 3, it is not easy to understand where such a serious error may lie but the possibility should obviously not be discounted.

\subsection{Solar neutrinos}

Neutrinos provide another means by which to infer the solar chemical composition, though current experiments are not yet sufficiently sensitive to return decisive results in this respect. The metallicity adopted in a solar model modifies the conditions in the core, and thus the neutrino fluxes produced by the pp-chain and CNO-cycle. Whilst the neutrino fluxes cannot be directly associated with abundances of individual elements, the relevant neutrino channels depend most sensitively on C, N, O, Si and Fe (Bahcall \& Serenelli 2005). Using neutrino data from the SuperKamiokande I+II, SNO and Borexino experiments, Pena-Garay \& Serenelli (2008) have derived the neutrino oscillations and fluxes expected from solar models constructed with the Grevesse \& Sauval (1998) and Asplund. Grevesse \& Sauval (2005) compositions. Intriguingly, the existing data imply ${ }^{7} \mathrm{Be}$ and ${ }^{8} \mathrm{~B}$ neutrino fluxes that fall between the predictions for the two models; we suspect that the solar neutrinos would favour the new abundances presented herein. A more direct test of the heavily debated solar C, N and $\mathrm{O}$ abundances would come from measuring the ${ }^{13} \mathrm{~N}$ and ${ }^{15} \mathrm{O} \beta$-decay neutrinos that follow proton capture on ${ }^{12} \mathrm{C}$ and ${ }^{14} \mathrm{~N}$, respectively. Meaningful constraints should come from Borexino within the next couple of years, and the proposed 
$\mathrm{SNO}+$ experiment should weigh in with a definite answer sometime after 2011 (Haxton \& Serenelli 2008).

\section{CONCLUDING REMARKS}

\subsection{Summary Points}

We have critically examined all ingredients for the determination of the solar photospheric chemical composition, from the atmospheric and line formation modelling, required atomic data and selection of lines to the final estimate of the elemental abundances and associated errors (Sect. 3). A notable feature of this analysis is that for essentially every element, the effects of 3D hydrodynamical model atmospheres have now been accounted for. The 3D solar model employed here outperforms any 1D model, theoretical or semi-empirical, in a raft of observational tests. The values we recommend for the most abundant metals $\mathrm{C}, \mathrm{N}, \mathrm{O}$, $\mathrm{Ne}$ and Fe are significantly smaller (by $\sim 0.2 \mathrm{dex}$ ) than those advocated in the widely-used compilation of Anders \& Grevesse (1989). Most of these differences can be attributed to the improvements over the past two decades in modelling the solar atmosphere, allowing for departures from LTE in the line formation, properly treating blends and employing improved atomic and molecular data (Sect. 2). Amongst other things, these new, lower abundances are supported by the excellent agreement between different abundance indicators for $\mathrm{C}, \mathrm{N}$ and $\mathrm{O}$ : low-excitation forbidden atomic lines, high-excitation permitted atomic lines and various molecular features.

A comparison of the photospheric abundances and the most primitive class of meteorites, the CI chondrites, reveals a very good agreement for nearly all nonvolatile elements (Sect. 4.1). Since the volatile elements, including H, have been depleted in the chondrites, the meteoritic abundance scale has been placed on the same absolute level as the photospheric abundances by enforcing equivalence of the $\mathrm{Si}$ abundances. The remaining few elements ( $\mathrm{Rb}, \mathrm{Rh}, \mathrm{Pd}, \mathrm{Ag}, \mathrm{Hf}, \mathrm{Pb}$ ) that suggest a significant difference between meteorites and the Sun can probably be attributed to poor solar determinations, likely due to incorrect $g f$-values, unidentified blends or unaccounted non-LTE effects.

In order to make a meaningful comparison between the Sun and the abundances measured in the solar neighborhood, one must first correct for the effects of diffusion in the Sun and chemical enrichment in the Galaxy over the past 4.56 Gyr. All elements are predicted to have settled to the solar interior by essentially the same amount, meaning that the bulk, or equivalently the proto-solar, composition is higher by 0.04 dex than listed in Table 1 for all elements heavier than $\mathrm{He}$ (Sect. 3.11). Our recommended solar abundances agree very well in general with those measured in nearby OB stars, H II regions like Orion and the local ISM

(Sect. 4.2). We note that care should be exercised when comparing our solar results with corresponding abundances for late-type stars derived with classical 1D model atmospheres; ideally a differential study of the stars and the Sun should be performed and using the solar abundances presented in Table 1 to place them on an absolute scale.

In spite of the many apparent successes of the solar chemical composition we recommend here, the outlook is not entirely rosy. With the downward revision of the overall metal content presented here compared to the Grevesse \& Sauval (1998) abundances, the previous excellent agreement between predicted sound 
speeds from standard solar models and those inferred from helioseismology is ruined (Sect. 4.3). To date there has been no fully convincing solution put forward. The discordance has been alleviated somewhat relative to the recommended values in Asplund, Grevesse \& Sauval (2005) but it nevertheless remains a significant discrepancy in urgent need of resolution.

\section{$5.2 \quad$ Future Issues}

While major progress has been made in recent years in the inferred solar chemical composition, there is no question that a great deal of work remains before we can confidently state that each solar elemental abundance is known to better than 0.01 dex $(2 \%)$. For many, if not most elements, the achieved accuracy today is more like 0.05 dex or indeed significantly worse in many cases. A concerted effort on multiple fronts is required to substantially decrease the remaining uncertainties.

We do not consider it likely that subsequent generations of 3D hydrodynamical model atmospheres will dramatically (i.e. at the $>0.1$ dex level) amend the inferred solar photospheric chemical composition, given how successful the most recent models are in reproducing key observational diagnostics. It is fair to say that the mean temperature structure of the quiet solar granulation, the velocity distribution and the atmospheric inhomogeneities are now rather well known, at least in the formation regions of the weaker lines largely employed by the abundance studies described herein. Higher atmospheric layers, to which the strongest atomic and molecular lines are more sensitive, are naturally less constrained observationally; on the modelling side this requires improved radiative transfer treatment and allowance of departures from LTE for key elements. For completeness, one should include the effects of magnetic fields in the 3D solar atmospheric models, but we do not expect that these will noticeably influence the derived abundances (Nordlund, Stein \& Asplund 2009).

The greatest uncertainties afflicting solar abundance work today instead stem from poor atomic and molecular data, and incomplete modelling of departures from LTE. Few spectral lines have their transition probabilities determined to better than a few \%. As an example here it suffices to mention the case of $\mathrm{Mg}$ with its relatively simple atomic structure: the majority of the $\mathrm{Mg}$ I lines employed here and elsewhere for solar abundance purposes still have quoted uncertainties of $25-50 \%$ according to the NIST database, which is clearly unacceptable. Fortunately there has been some laudable progress on this score in recent years, both in terms of experimental efforts (e.g. Blackwell-Whitehead \& Bergemann 2007, Johansson et al. 2003, Lawler et al. 2009), and theoretical calculations (e.g. Badnell et al. 2005, Kurucz 1992, Plez 1998) as discussed in Sect. 2.2. A major problem, however, is the dearth of atomic physicists devoted to satisfying the astronomical need for various types of input data; the astronomical community ought to better appreciate and encourage the relatively few individuals carrying out this important work, given its tremendous benefit for solar and stellar astrophysics especially. On the wish-list are improved $g f$-values for $\mathrm{Mg}$ I, $\mathrm{Mg}$ II, Si II, Ca II, the once-ionized species of the Fe-peak elements, Cu I and Sr II. Also, atomic lines in the IR have great potential given the accurate determination of the continuum and few blends but more often than not accurate transition probabilities are lacking.

Equally important is the consideration of non-LTE effects on line formation. 
As discussed by Asplund (2005), even though departures from LTE are expected to be relatively serene in the Sun, one should always expect non-LTE abundance corrections at around the 0.05 dex level; indeed in many cases the effects will be significantly more severe. Still today, most elements have not been exposed to a detailed non-LTE study even in the Sun. Even rarer are solar abundance works going beyond the 1D framework and employing 3D non-LTE calculations (e.g. Asplund, Carlsson \& Botnen 2003, Asplund et al. 2004). In part, the scarcity of non-LTE studies is related to a lack of adequate atomic data. Compared with the LTE case, vastly more input data are required in non-LTE calculations than are presently available for most elements. Significant headway will only become possible with the advent of detailed photo-ionization and collisional cross-sections; for the latter, estimates of the impact of both electron and hydrogen collisions, as well as other processes like charge transfer, are urgently needed. There is also an issue of manpower; careful non-LTE studies are very time-consuming and there are not enough specialists working in the field to satisfy the demand. Finally, we note that non-LTE line formation should be explored in 3D model atmospheres, since the the presence of atmospheric inhomogeneities is generally expected to amplify any existing non-LTE effects seen in 1D.

A section on future issues in the solar chemical composition would not be complete without stressing the importance of finally resolving the solar modelling problem uncovered by helioseismology (Sect. 4.3). Many hypotheses have been put forward to explain the discrepancy, but no clear solution has been forthcoming. Until an explanation has been found, a mutual cloud of doubt will always linger over the solar chemical composition recommended here and current models of the solar interior. Whether the problem will be traced in the end to shortcomings in the atmospheric or interior modelling remains to be seen, but whatever the resolution it will place solar and stellar astrophysics - and by extension most of astronomy - on a much firmer footing.

\subsection{Disclosure Statement}

The authors are not aware of any affiliations, memberships, funding, or financial holdings that might be perceived as affecting the objectivity of this review.

\subsection{Acknowledgements}

We are indebted to our collaborators over the years for their important contribution to uncovering the solar chemical composition. We would particularly like to highlight the contributions of Carlos Allende Prieto, David Lambert, Jorge Meléndez and Tiago Pereira. The work presented here would not have been possible without extensive efforts in solar atmospheric and line-formation modelling by our colleagues Mats Carlsson, Remo Collet, Wolfgang Hayek, Dan Kiselman, Åke Nordlund, Bob Stein and Regner Trampedach. We thank Tiago Pereira and Aldo Serenelli for providing figures. Finally, we acknowledge a large number of colleagues around the world for many stimulating discussions, healthy and friendly competition, providing information prior to publication and invaluable comments on the manuscript and topics discussed herein. 


\section{References}

Abia C, Mashonkina L. 2004. MNRAS 350:1127-1140

Abrams MC, Goldman A, Gunson MR, Rinsland CP, Zander R. 1996. Applied Optics 35:2747-2751

Allard NF, Kielkopf JF, Cayrel R, van't Veer-Menneret C. 2008. Astron. Astrophys. 480:581-587

Allende Prieto C, Asplund M, Fabiani Bendicho P. 2004. Astron. Astrophys. 423:1109-1117

Allende Prieto C, Barklem PS, Asplund M, Ruiz Cobo B. 2001. Ap. J. 558:830851

Allende Prieto C, Lambert DL, Asplund M. 2001. Ap. J. L. 556:L63-L66

Allende Prieto C, Lambert DL, Asplund M. 2002. Ap. J. L. 573:L137-L140

Anders E, Grevesse N. 1989. Geochim. Cosmochim. Acta 53:197-214

Anstee SD, O'Mara BJ. 1995. MNRAS 276:859-866

Anstee SD, O'Mara BJ, Ross JE. 1997. MNRAS 284:202-212

Antia HM, Basu S. 2005. Ap. J. L. 620:L129-L132

Arnett D, Meakin C, Young PA. 2005. In Cosmic Abundances as Records of Stellar Evolution and Nucleosynthesis, eds. TG Barnes III, FN Bash, vol. 336 of Astronomical Society of the Pacific Conference Series

Asplund M. 2000. Astron. Astrophys. 359:755-758

Asplund M. 2004. Astron. Astrophys. 417:769-774

Asplund M. 2005. Annu. Rev. Astron. Astrophys. 43:481-530

Asplund M, Carlsson M, Botnen AV. 2003. Astron. Astrophys. 399:L31-L34

Asplund M, Grevesse N, Sauval AJ. 2005. In Cosmic Abundances as Records of Stellar Evolution and Nucleosynthesis, eds. TG Barnes III, FN Bash, vol. 336 of Astronomical Society of the Pacific Conference Series

Asplund M, Grevesse N, Sauval AJ, Allende Prieto C, Blomme R. 2005. Astron. Astrophys. 431:693-705

Asplund M, Grevesse N, Sauval AJ, Allende Prieto C, Kiselman D. 2004. Astron. Astrophys. 417:751-768

Asplund M, Grevesse N, Sauval AJ, Scott P, Trampedach R. 2009a. Astron. Astrophys. to be submitted

Asplund M, Grevesse N, Sauval AJ, Scott P, Trampedach R. 2009b. Astron. Astrophys. to be submitted

Asplund M, Ludwig HG, Nordlund A, Stein RF. 2000a. Astron. Astrophys. 359:669-681

Asplund M, Nordlund A, Trampedach R, Allende Prieto C, Stein RF. 2000b. Astron. Astrophys. 359:729-742

Asplund M, Nordlund A, Trampedach R, Stein RF. 1999. Astron. Astrophys. 346:L17-L20

Asplund M, Nordlund A, Trampedach R, Stein RF. 2000c. Astron. Astrophys. 359:743-754 
Asplund M, Pereira T, Grevesse N, Sauval AJ, Scott P, Trampedach R. 2009c. Astron. Astrophys. to be submitted

Ayres TR. 2008. Ap. J. 686:731-740

Ayres TR, Plymate C, Keller CU. 2006. Ap. J. Supp. 165:618-651

Badnell NR, Bautista MA, Butler K, Delahaye F, Mendoza C, et al. 2005. MNRAS 360:458-464

Bahcall JN, Basu S, Pinsonneault M, Serenelli AM. 2005. Ap. J. 618:1049-1056

Bahcall JN, Basu S, Serenelli AM. 2005. Ap. J. 631:1281-1285

Bahcall JN, Serenelli AM. 2005. Ap. J. 626:530-542

Balachandran SC, Bell RA. 1998. Nature 392:791-793

Barklem PS. 2007a. Astron. Astrophys. 462:781-788

Barklem PS. 2007b. Astron. Astrophys. 466:327-337

Barklem PS, Aspelund-Johansson J. 2005. Astron. Astrophys. 435:373-377

Barklem PS, Belyaev AK, Asplund M. 2003. Astron. Astrophys. 409:L1-L4

Barklem PS, O’Mara BJ. 2000. MNRAS 311:535-540

Barklem PS, Piskunov N, O'Mara BJ. 2000a. Astron. Astrophys. Supp. 142:467473

Barklem PS, Piskunov N, O'Mara BJ. 2000b. Astron. Astrophys. 363:1091-1105

Barklem PS, Stempels HC, Allende Prieto C, Kochukhov OP, Piskunov N, O'Mara BJ. 2002. Astron. Astrophys. 385:951-967

Basu S, Antia HM. 2004. Ap. J. L. 606:L85-L88

Basu S, Antia HM. 2008. Physics Reports 457:217-283

Bell RA, Balachandran SC, Bautista M. 2001. Ap. J. L. 546:L65-L68

Belyaev AK, Barklem PS. 2003. Phys. Rev. A 68:062703-062711

Bensby T, Feltzing S, Lundström I, Ilyin I. 2005. Astron. Astrophys. 433:185-203

Bergemann M. 2008. Physica Scripta Volume T 133:014013-+

Bergemann M, Gehren T. 2007. Astron. Astrophys. 473:291-302

Biémont E, Garnir HP, Palmeri P, Li ZS, Svanberg S. 2000. MNRAS 312:116-122

Biémont E, Lyngå C, Li ZS, Svanberg S, Garnir HP, Doidge PS. 1999. MNRAS 303:721-726

Blackwell DE, Lynas-Gray AE, Smith G. 1995. Astron. Astrophys. 296:217-232

Blackwell-Whitehead R, Bergemann M. 2007. Astron. Astrophys. 472:L43-L46

Bochsler P. 2007. Astron. Astrophys. 471:315-319

Böhm-Vitense E. 1958. Zeitschrift fur Astrophysik 46:108-143

Boothroyd AI, Sackmann IJ. 2003. Ap. J. 583:1004-1023

Brun AS, Turck-Chièze S, Zahn JP. 1999. Ap. J. 525:1032-1041

Caffau E, Faraggiana R, Bonifacio P, Ludwig HG, Steffen M. 2007a. Astron. Astrophys. 470:699-708

Caffau E, Ludwig HG. 2007. Astron. Astrophys. 467:L11-L14 
Caffau E, Ludwig HG, Steffen M, Ayres TR, Bonifacio P, et al. 2008a. Astron. Astrophys. 488:1031-1046

Caffau E, Maiorca E, Bonifacio P, Faraggiana R, Steffen M, et al. 2009. Astron. Astrophys. 498:877-884

Caffau E, Sbordone L, Ludwig HG, Bonifacio P, Steffen M, Behara NT. 2008b. Astron. Astrophys. 483:591-598

Caffau E, Steffen M, Sbordone L, Ludwig HG, Bonifacio P. 2007b. Astron. Astrophys. 473:L9-L12

Cartledge SIB, Lauroesch JT, Meyer DM, Sofia UJ. 2006. Ap. J. 641:327-346

Castro M, Vauclair S, Richard O. 2007. Astron. Astrophys. 463:755-758

Centeno R, Socas-Navarro H. 2008. Ap. J. L. 682:L61-L64

Chaplin WJ, Serenelli AM, Basu S, Elsworth Y, New R, Verner GA. 2007. Ap. J. $670: 872-884$

Charbonnel C, Talon S. 2005. Science 309:2189-2191

Chiappini C, Romano D, Matteucci F. 2003. MNRAS 339:63-81

Chmielewski Y, Brault JW, Müller EA. 1975. Astron. Astrophys. 42:37-46

Christensen-Dalsgaard J, di Mauro MP, Houdek G, Pijpers F. 2009. Astron. Astrophys. 494:205-208

Collet R, Asplund M, Thévenin F. 2005. Astron. Astrophys. 442:643-650

Coplen T, Böhlke J, De Bièvre P, et al. 2002. Pure ES Appl. Chem. 74:1987-2017

Cunha K, Smith VV. 1999. Ap. J. 512:1006-1013

Daflon S, Cunha K. 2004. Ap. J. 617:1115-1126

Delahaye F, Pinsonneault MH. 2006. Ap. J. 649:529-540

Delbouille L, Roland G, Brault J, Testerman L. 1981. Photometric atlas of the solar spectrum from 1850 to $10000 \mathrm{~cm}^{-1}$. Institut d'Astrophysique, Université de Liège (http://bass2000.obspm.fr/solar_spect.php)

Delbouille L, Roland G, Neven L. 1973. Photometric atlas of the solar spectrum from 3000 to $10000 \AA$. Institut d'Astrophysique, Université de Liège (http://bass2000.obspm.fr/solar_spect.php)

Den Hartog EA, Lawler JE, Sneden C, Cowan JJ. 2003. Ap. J. Supp. 148:543-566

Den Hartog EA, Lawler JE, Sneden C, Cowan JJ. 2006. Ap. J. Supp. 167:292-314

Drake JJ, Testa P. 2005. Nature 436:525-528

Drawin HW. 1968. Zeitschrift fur Physik 211:404-417

Edvardsson B, Andersen J, Gustafsson B, Lambert DL, Nissen PE, Tomkin J. 1993. Astron. Astrophys. 275:101-152

Esteban C, García-Rojas J, Peimbert M, Peimbert A, Ruiz MT, et al. 2005. Ap. J. L. 618:L95-L98

Esteban C, Peimbert M, García-Rojas J, Ruiz MT, Peimbert A, Rodríguez M. 2004. MNRAS 355:229-247

Fabbian D, Asplund M, Barklem PS, Carlsson M, Kiselman D. 2009. Astron. Astrophys. 500:1221-1238 
Fabbian D, Asplund M, Carlsson M, Kiselman D. 2006. Astron. Astrophys. 458:899-914

Farmer C, Norton R. 1989. NASA Reference Publication 1224 (http://remus.jpl.nasa.gov/atmos/ftp.at3.sun.html)

Feldman U, Widing KG. 2003. Space Science Reviews 107:665-720

Fivet V, Quinet P, Palmeri P, Biémont É, Asplund M, et al. 2009. MNRAS 396:2124-2132

Fontenla JM, Avrett E, Thuillier G, Harder J. 2006. Ap. J. 639:441-458

Freytag B, Steffen M, Dorch B. 2002. Astronomische Nachrichten 323:213-219

Fuhrmann K. 2008. MNRAS 384:173-224

García RA, Turck-Chièze S, Jiménez-Reyes SJ, Ballot J, Pallé PL, et al. 2007. Science 316:1591-1593

García-Rojas J, Esteban C. 2007. Ap. J. 670:457-470

Gehren T, Liang YC, Shi JR, Zhang HW, Zhao G. 2004. Astron. Astrophys. 413:1045-1063

Geiss J, Gloeckler G, von Steiger R. 1994. Royal Society of London Philosophical Transactions Series A 349:213-226

Gloeckler G, Geiss J. 2000. In The Light Elements and their Evolution, eds. L da Silva, R de Medeiros, M Spite, vol. 198 of IAU Symposium

Goldberg L, Müller EA, Aller LH. 1960. Ap. J. Supp. 5:1-137

Gopka VF, Yushchenko AV, Shavrina AV, Andrievsky SM, Chernysheva LV. 2001. Kinematika i Fizika Nebesnykh Tel 17:37-44

Grevesse N, Asplund M, Sauval AJ. 2007. Space Science Reviews 130:105-114

Grevesse N, Asplund M, Sauval AJ, Scott P, Trampedach R. 2009. Astron. Astrophys. to be submitted

Grevesse N, Lambert DL, Sauval AJ, van Dishoeck EF, Farmer CB, Norton RH. 1990. Astron. Astrophys. 232:225-230

Grevesse N, Lambert DL, Sauval AJ, van Dishoek EF, Farmer CB, Norton RH. 1991. Astron. Astrophys. 242:488-495

Grevesse N, Noels A. 1993. In Origin and Evolution of the Elements, eds. N Prantzos, E Vangioni-Flam, M Cassé

Grevesse N, Sauval AJ. 1998. Space Science Reviews 85:161-174

Grevesse N, Sauval AJ. 1999. Astron. Astrophys. 347:348-354

Grevesse N, Sauval AJ, van Dishoeck EF. 1984. Astron. Astrophys. 141:10-16

Güdel M. 2004. Astron. Astrophys. Review 12:71-237

Gustafsson B. 2008. Physica Scripta Volume T 130:014036-014041

Gustafsson B, Edvardsson B, Eriksson K, Jørgensen UG, Nordlund A, Plez B. 2008. Astron. Astrophys. 486:951-970

Guzik JA, Watson LS, Cox AN. 2005. Ap. J. 627:1049-1056

Hall DNB, Noyes RW. 1969. Astroph. Letters. 4:143-148

Hall DNB, Noyes RW. 1972. Ap. J. L. 175:L95-L97 
Hannaford P, Lowe RM, Grevesse N, Noels A. 1992. Astron. Astrophys. 259:301306

Hashizume K, Chaussidon M. 2005. Nature 434:619-622

Haxton WC, Serenelli AM. 2008. Ap. J. 687:678-691

Heber VS, Baur H, Bochsler P, Burnett DS, Reisenfeld DB, et al. 2008a. In Lunar and Planetary Institute Conference Abstracts, vol. 39 of Lunar and Planetary Inst. Technical Report

Heber VS, Baur H, Wieler R, Vogel N, Wiens RC, Burnett DS. 2008b. AGU Fall Meeting Abstracts :A8

Holmberg J, Nordström B, Andersen J. 2008. Astron. Astrophys. in press (arXiv: 0811.3982)

Holweger H. 1967. Zeitschrift fur Astrophysik 65:365-417

Holweger H. 2001. In Joint SOHO/ACE workshop "Solar and Galactic Composition", ed. RF Wimmer-Schweingruber, vol. 598 of American Institute of Physics Conference Series

Holweger H, Kock M, Bard A. 1995. Astron. Astrophys. 296:233-240

Holweger H, Müller EA. 1974. Solar Physics 39:19-30

Holweger H, Werner K. 1982. Solar Physics 81:3-8

Ireland TR, Holden P, Norman MD, Clarke J. 2006. Nature 440:776-778

Jenkins EB. 2004. In Origin and Evolution of the Elements, eds. A McWilliam, M Rauch

Jensen AG. 2007. Updated interstellar abundance studies and implications for dust models. Ph.D. thesis, University of Colorado at Boulder

Jensen AG, Rachford BL, Snow TP. 2005. Ap. J. 619:891-913

Jensen AG, Rachford BL, Snow TP. 2007. Ap. J. 654:955-970

Johansson S, Litzén U, Lundberg H, Zhang Z. 2003. Ap. J. L. 584:L107-L110

Käppeler F. 2005. In Cosmic Abundances as Records of Stellar Evolution and Nucleosynthesis, eds. TG Barnes III, FN Bash, vol. 336 of Astronomical Society of the Pacific Conference Series

Kiselman D. 1993. Astron. Astrophys. 275:269-282

Kiselman D. 1997. Ap. J. L. 489:L107-L110

Kiselman D, Carlsson M. 1996. Astron. Astrophys. 311:680-689

Koesterke L, Allende Prieto C, Lambert DL. 2008. Ap. J. 680:764-773

Korn AJ, Shi J, Gehren T. 2003. Astron. Astrophys. 407:691-703

Kurucz RL. 1992. Revista Mexicana de Astronomia y Astrofisica, vol. 23 23:4548

Kurucz RL. 1993. In IAU Colloq. 138: Peculiar versus Normal Phenomena in Atype and Related Stars, eds. MM Dworetsky, F Castelli, R Faraggiana, vol. 44 of Astronomical Society of the Pacific Conference Series

Kurucz RL. 2006. arXiv: astro-ph/0605029

Kurucz RL, Furenlid I, Brault J, Testerman L. 1984. Solar flux atlas from 296 to $1300 \mathrm{~nm}$ 
Lambert DL. 1978. MNRAS 182:249-271

Lambert DL, Luck RE. 1978. MNRAS 183:79-100

Lambert DL, Mallia EA, Smith G. 1972. Solar Physics 26:250-256

Landi E, Feldman U, Doschek GA. 2007. Ap. J. 659:743-749

Lanz T, Cunha K, Holtzman J, Hubeny I. 2008. Ap. J. 678:1342-1350

Lawler JE, Bonvallet G, Sneden C. 2001. Ap. J. 556:452-460

Lawler JE, den Hartog EA, Labby ZE, Sneden C, Cowan JJ, Ivans II. 2007. Ap. J. Supp. 169:120-136

Lawler JE, Den Hartog EA, Sneden C, Cowan JJ. 2006. Ap. J. Supp. 162:227-260

Lawler JE, Sneden C, Cowan JJ. 2004. Ap. J. 604:850-860

Lawler JE, Sneden C, Cowan JJ, Ivans II, Den Hartog EA. 2009. Ap. J. Supp. 182:51-79

Lawler JE, Sneden C, Cowan JJ, Wyart JF, Ivans II, et al. 2008. Ap. J. Supp. 178:71-88

Lawler JE, Wickliffe ME, Cowley CR, Sneden C. 2001a. Ap. J. Supp. 137:341-349

Lawler JE, Wickliffe ME, den Hartog EA, Sneden C. 2001b. Ap. J. 563:1075-1088

Lellouch E, Bézard B, Fouchet T, Feuchtgruber H, Encrenaz T, de Graauw T. 2001. Astron. Astrophys. 370:610-622

Linsky JL, Draine BT, Moos HW, Jenkins EB, Wood BE, et al. 2006. Ap. J. 647:1106-1124

Ljung G, Nilsson H, Asplund M, Johansson S. 2006. Astron. Astrophys. 456:11811185

Lodders K. 2003. Ap. J. 591:1220-1247

Lodders K. 2008. Ap. J. 674:607-611

Lodders K, Palme H, Gail H. 2009. Landolt-Börnstein series in Astronomy and Astrophysics submitted (arXiv: 0901.1149)

Mahaffy PR, Donahue TM, Atreya SK, Owen TC, Niemann HB. 1998. Space Science Reviews 84:251-263

Mashonkina L, Gehren T. 2000. Astron. Astrophys. 364:249-264

Mashonkina L, Gehren T. 2001. Astron. Astrophys. 376:232-247

Mashonkina L, Korn AJ, Przybilla N. 2007. Astron. Astrophys. 461:261-275

McKeegan KD, Coath CD, Heber V, Jarzebinski G, Kallio A, et al. 2008. AGU Fall Meeting Abstracts :42A-07

Meibom A, Krot AN, Robert F, Mostefaoui S, Russell SS, et al. 2007. Ap. J. L. 656:L33-L36

Meléndez J. 2004. Ap. J. 615:1042-1047

Meléndez J, Asplund M. 2008. Astron. Astrophys. 490:817-821

Meléndez J, Asplund M, Gustafsson B, Yong D. 2009. Ap. J. L. in press

Meléndez J, Barbuy B. 2009. Astron. Astrophys. 497:611-617

Mihalas D, Däppen W, Hummer DG. 1988. Ap. J. 331:815-825 
Montalbán J, Miglio A, Noels A, Grevesse N, di Mauro MP. 2004. In SOHO 14 Helio- and Asteroseismology: Towards a Golden Future, ed. D Danesy, vol. 559 of ESA Special Publication

Morel T. 2008. Comm. in Asteroseismology in press (arXiv: 0811.4114)

Morel T, Butler K, Aerts C, Neiner C, Briquet M. 2006. Astron. Astrophys. 457:651-663

Mucciarelli A, Caffau E, Freytag B, Ludwig HG, Bonifacio P. 2008. Astron. Astrophys. 484:841-845

Müller EA, Peytremann E, de La Reza R. 1975. Solar Physics 41:53-65

Neckel H, Labs D. 1984. Solar Physics 90:205-258

Neckel H, Labs D. 1994. Solar Physics 153:91-114

Nieva MF, Przybilla N. 2006. Ap. J. L. 639:L39-L42

Nordlund A. 1982. Astron. Astrophys. 107:1-10

Nordlund A, Stein RF, Asplund M. 2009. Living Reviews in Solar Physics 6:2-+

Owen T, Mahaffy PR, Niemann HB, Atreya S, Wong M. 2001. Ap. J. L. 553:L77L79

Pagel BEJ. 1997. Nucleosynthesis and Chemical Evolution of Galaxies

Pena-Garay C, Serenelli A. 2008. arXiv: 0811.2424

Pereira T, Asplund M, Kiselman D. 2009. Astron. Astrophys. submitted

Pereira T, Asplund M, Trampedach R, Collet R. 2009. Astron. Astrophys. submitted

Plez B. 1998. Astron. Astrophys. 337:495-500

Prantzos N. 2008. In EAS Publications Series, eds. C Charbonnel, JP Zahn, vol. 32 of EAS Publications Series

Przybilla N, Nieva MF, Butler K. 2008. Ap. J. L. 688:L103-L106

Quinet P, Palmeri P, Biémont É, Jorissen A, van Eck S, et al. 2006. Astron. Astrophys. 448:1207-1216

Ramírez I, Allende Prieto C, Lambert DL. 2007. Astron. Astrophys. 465:271-289

Reames DV. 1999. Space Science Reviews 90:413-491

Reddy BE, Tomkin J, Lambert DL, Allende Prieto C. 2003. MNRAS 340:304-340

Robles JA, Lineweaver CH, Grether D, Flynn C, Egan CA, et al. 2008. Ap. J. 684:691-706

Robrade J, Schmitt JHMM, Favata F. 2008. Astron. Astrophys. 486:995-1002

Rogers FJ, Nayfonov A. 2002. Ap. J. 576:1064-1074

Rosman KJR, Taylor PDP. 1998. Journal of Physical and Chemical Reference Data 27:1275-1287

Ross JE, Aller LH. 1976. Science 191:1223-1229

Russell HN. 1929. Ap. J. 70:11-82

Sauval AJ, Asplund M, Grevesse N, van Hemert M, Blomme R, Trampedach R. 2009. Astron. Astrophys. to be submitted 
Sauval AJ, Grevesse N, Zander R, Brault JW, Stokes GM. 1984. Ap. J. 282:330338

Schmelz JT, Nasraoui K, Roames JK, Lippner LA, Garst JW. 2005. Ap. J. L. 634:L197-L200

Schnabel R, Kock M, Holweger H. 1999. Astron. Astrophys. 342:610-613

Scott P, Asplund M, Grevesse N, Sauval AJ. 2009a. Ap. J. L. 691:L119-L122

Scott P, Asplund M, Grevesse N, Sauval AJ, Trampedach R. 2009b. Astron. Astrophys. to be submitted

Scott PC, Asplund M, Grevesse N, Sauval AJ. 2006. Astron. Astrophys. 456:675688

Serenelli AM, Basu S, Ferguson JW, Asplund M. 2009. Ap. J. L. to be submitted

Shi JR, Gehren T, Butler K, Mashonkina LI, Zhao G. 2008. Astron. Astrophys. 486:303-310

Shi JR, Gehren T, Zhao G. 2004. Astron. Astrophys. 423:683-691

Short CI, Hauschildt PH. 2005. Ap. J. 618:926-938

Sneden C, Lawler JE, Cowan JJ, Ivans II, Den Hartog EA. 2009. Ap. J. Supp. 182:80-96

Sobeck JS, Lawler JE, Sneden C. 2007. Ap. J. 667:1267-1282

Sofia UJ, Lauroesch JT, Meyer DM, Cartledge SIB. 2004. Ap. J. 605:272-277

Sofia UJ, Meyer DM. 2001. Ap. J. L. 554:L221-L224

Stein RF, Nordlund A. 1998. Ap. J. 499:914-933

Suess HE, Urey HC. 1956. Reviews of Modern Physics 28:53-74

Takeda Y, Hashimoto O, Taguchi H, Yoshioka K, Takada-Hidai M, et al. 2005. P. A.S.J. 57:751-768

Takeda Y, Zhao G, Takada-Hidai M, Chen YQ, Saito YJ, Zhang HW. 2003. Chinese Journal of Astronomy and Astrophysics 3:316-340

Trampedach R, Asplund M, Hayek W, Collet R. 2009. Astron. Astrophys. to be submitted

Trampedach R, Däppen W, Baturin VA. 2006. Ap. J. 646:560-578

Turck-Chièze S, Couvidat S, Piau L, Ferguson J, Lambert P, et al. 2004. Physical Review Letters 93:211102

Turcotte S, Wimmer-Schweingruber RF. 2002. Journal of Geophysical Research (Space Physics) 107:1442-1451

van Regemorter H. 1962. Ap. J. 136:906-915

Vernazza JE, Avrett EH, Loeser R. 1976. Ap. J. Supp. 30:1-60

Vitas N, Vince I, Lugaro M, Andriyenko O, Gošić M, Rutten RJ. 2008. MNRAS 384:370-375

Vögler A, Bruls JHMJ, Schüssler M. 2004. Astron. Astrophys. 421:741-754

Wieler R. 2002. Rev. Mineral. Geochim. 47:21-70

Xu HL, Sun ZW, Dai ZW, Jiang ZK, Palmeri P, et al. 2006. Astron. Astrophys. 452:357-362 
Yang WM, Bi SL. 2007. Ap. J. L. 658:L67-L70

Young PA, Arnett D. 2005. Ap. J. 618:908-918

Young PR. 2005a. Astron. Astrophys. 439:361-366

Young PR. 2005b. Astron. Astrophys. 444:L45-L48

Youssef NH. 1986. Astron. Astrophys. 164:395-396

Youssef NH, Doenszelmann A, Grevesse N. 1990. Astron. Astrophys. 239:367-368

Youssef NH, Khalil NM. 1988. Astron. Astrophys. 203:378-380

Zhang HW, Butler K, Gehren T, Shi JR, Zhao G. 2006. Astron. Astrophys. 453:723-727

Zhang HW, Gehren T, Zhao G. 2008. Astron. Astrophys. 481:489-497

Zhao G, Butler K, Gehren T. 1998. Astron. Astrophys. 333:219-230 
Table 1: Element abundances in the present-day solar photosphere. Also given are the corresponding values for CI carbonaceous chondrites (Lodders, Palme \& Gail 2009). Indirect photospheric estimates have been used for the noble gases (Sect. 3.9).

\begin{tabular}{|c|c|c|c|c|c|c|c|}
\hline & Elem. & Photosphere & Meteorites & & Elem. & Photosphere & Meteorites \\
\hline 1 & $\mathrm{H}$ & 12.00 & $8.22 \pm 0.04$ & 44 & $\mathrm{Ru}$ & $1.75 \pm 0.08$ & $1.76 \pm 0.03$ \\
\hline 2 & $\mathrm{He}$ & {$[10.93 \pm 0.01]$} & 1.29 & 45 & $\mathrm{Rh}$ & $0.91 \pm 0.10$ & $1.06 \pm 0.04$ \\
\hline 3 & $\mathrm{Li}$ & $1.05 \pm 0.10$ & $3.26 \pm 0.05$ & 46 & $\mathrm{Pd}$ & $1.57 \pm 0.10$ & $1.65 \pm 0.02$ \\
\hline 4 & $\mathrm{Be}$ & $1.38 \pm 0.09$ & $1.30 \pm 0.03$ & 47 & $\mathrm{Ag}$ & $0.94 \pm 0.10$ & $1.20 \pm 0.02$ \\
\hline 5 & $\mathrm{~B}$ & $2.70 \pm 0.20$ & $2.79 \pm 0.04$ & 48 & $\mathrm{Cd}$ & & $1.71 \pm 0.03$ \\
\hline 6 & $\mathrm{C}$ & $8.43 \pm 0.05$ & $7.39 \pm 0.04$ & 49 & In & $0.80 \pm 0.20$ & $0.76 \pm 0.03$ \\
\hline 7 & $\mathrm{~N}$ & $7.83 \pm 0.05$ & $6.26 \pm 0.06$ & 50 & Sn & $2.04 \pm 0.10$ & $2.07 \pm 0.06$ \\
\hline 8 & $\mathrm{O}$ & $8.69 \pm 0.05$ & $8.40 \pm 0.04$ & 51 & $\mathrm{Sb}$ & & $1.01 \pm 0.06$ \\
\hline 9 & $\mathrm{~F}$ & $4.56 \pm 0.30$ & $4.42 \pm 0.06$ & 52 & $\mathrm{Te}$ & & $2.18 \pm 0.03$ \\
\hline 10 & $\mathrm{Ne}$ & {$[7.93 \pm 0.10]$} & -1.12 & 53 & I & & $1.55 \pm 0.08$ \\
\hline 11 & $\mathrm{Na}$ & $6.24 \pm 0.04$ & $6.27 \pm 0.02$ & 54 & $\mathrm{Xe}$ & {$[2.24 \pm 0.06]$} & -1.95 \\
\hline 12 & $\mathrm{Mg}$ & $7.60 \pm 0.04$ & $7.53 \pm 0.01$ & 55 & Cs & & $1.08 \pm 0.02$ \\
\hline 13 & $\mathrm{Al}$ & $6.45 \pm 0.03$ & $6.43 \pm 0.01$ & 56 & $\mathrm{Ba}$ & $2.18 \pm 0.09$ & $2.18 \pm 0.03$ \\
\hline 14 & $\mathrm{Si}$ & $7.51 \pm 0.03$ & $7.51 \pm 0.01$ & 57 & $\mathrm{La}$ & $1.10 \pm 0.04$ & $1.17 \pm 0.02$ \\
\hline 15 & $\mathrm{P}$ & $5.41 \pm 0.03$ & $5.43 \pm 0.04$ & 58 & $\mathrm{Ce}$ & $1.58 \pm 0.04$ & $1.58 \pm 0.02$ \\
\hline 16 & $\mathrm{~S}$ & $7.12 \pm 0.03$ & $7.15 \pm 0.02$ & 59 & $\operatorname{Pr}$ & $0.72 \pm 0.04$ & $0.76 \pm 0.03$ \\
\hline 17 & $\mathrm{Cl}$ & $5.50 \pm 0.30$ & $5.23 \pm 0.06$ & 60 & $\mathrm{Nd}$ & $1.42 \pm 0.04$ & $1.45 \pm 0.02$ \\
\hline 18 & $\mathrm{Ar}$ & {$[6.40 \pm 0.13]$} & -0.50 & 62 & $\mathrm{Sm}$ & $0.96 \pm 0.04$ & $0.94 \pm 0.02$ \\
\hline 19 & $\mathrm{~K}$ & $5.03 \pm 0.09$ & $5.08 \pm 0.02$ & 63 & $\mathrm{Eu}$ & $0.52 \pm 0.04$ & $0.51 \pm 0.02$ \\
\hline 20 & $\mathrm{Ca}$ & $6.34 \pm 0.04$ & $6.29 \pm 0.02$ & 64 & $\mathrm{Gd}$ & $1.07 \pm 0.04$ & $1.05 \pm 0.02$ \\
\hline 21 & $\mathrm{Sc}$ & $3.15 \pm 0.04$ & $3.05 \pm 0.02$ & 65 & $\mathrm{~Tb}$ & $0.30 \pm 0.10$ & $0.32 \pm 0.03$ \\
\hline 22 & $\mathrm{Ti}$ & $4.95 \pm 0.05$ & $4.91 \pm 0.03$ & 66 & Dy & $1.10 \pm 0.04$ & $1.13 \pm 0.02$ \\
\hline 23 & $\mathrm{~V}$ & $3.93 \pm 0.08$ & $3.96 \pm 0.02$ & 67 & Ho & $0.48 \pm 0.11$ & $0.47 \pm 0.03$ \\
\hline 24 & $\mathrm{Cr}$ & $5.64 \pm 0.04$ & $5.64 \pm 0.01$ & 68 & Er & $0.92 \pm 0.05$ & $0.92 \pm 0.02$ \\
\hline 25 & $\mathrm{Mn}$ & $5.43 \pm 0.05$ & $5.48 \pm 0.01$ & 69 & $\mathrm{Tm}$ & $0.10 \pm 0.04$ & $0.12 \pm 0.03$ \\
\hline 26 & $\mathrm{Fe}$ & $7.50 \pm 0.04$ & $7.45 \pm 0.01$ & 70 & $\mathrm{Yb}$ & $0.84 \pm 0.11$ & $0.92 \pm 0.02$ \\
\hline 27 & $\mathrm{Co}$ & $4.99 \pm 0.07$ & $4.87 \pm 0.01$ & 71 & $\mathrm{Lu}$ & $0.10 \pm 0.09$ & $0.09 \pm 0.02$ \\
\hline 28 & $\mathrm{Ni}$ & $6.22 \pm 0.04$ & $6.20 \pm 0.01$ & 72 & Hf & $0.85 \pm 0.04$ & $0.71 \pm 0.02$ \\
\hline 29 & $\mathrm{Cu}$ & $4.19 \pm 0.04$ & $4.25 \pm 0.04$ & 73 & $\mathrm{Ta}$ & & $-0.12 \pm 0.04$ \\
\hline 30 & $\mathrm{Zn}$ & $4.56 \pm 0.05$ & $4.63 \pm 0.04$ & 74 & $\mathrm{~W}$ & $0.85 \pm 0.12$ & $0.65 \pm 0.04$ \\
\hline 31 & $\mathrm{Ga}$ & $3.04 \pm 0.09$ & $3.08 \pm 0.02$ & 75 & $\operatorname{Re}$ & & $0.26 \pm 0.04$ \\
\hline 32 & $\mathrm{Ge}$ & $3.65 \pm 0.10$ & $3.58 \pm 0.04$ & 76 & Os & $1.40 \pm 0.08$ & $1.35 \pm 0.03$ \\
\hline 33 & As & & $2.30 \pm 0.04$ & 77 & $\mathrm{Ir}$ & $1.38 \pm 0.07$ & $1.32 \pm 0.02$ \\
\hline 34 & $\mathrm{Se}$ & & $3.34 \pm 0.03$ & 78 & $\mathrm{Pt}$ & & $1.62 \pm 0.03$ \\
\hline 35 & $\mathrm{Br}$ & & $2.54 \pm 0.06$ & 79 & $\mathrm{Au}$ & $0.92 \pm 0.10$ & $0.80 \pm 0.04$ \\
\hline 36 & $\mathrm{Kr}$ & {$[3.25 \pm 0.06]$} & -2.27 & 80 & $\mathrm{Hg}$ & & $1.17 \pm 0.08$ \\
\hline 37 & $\mathrm{Rb}$ & $2.52 \pm 0.10$ & $2.36 \pm 0.03$ & 81 & $\mathrm{Tl}$ & $0.90 \pm 0.20$ & $0.77 \pm 0.03$ \\
\hline 38 & $\mathrm{Sr}$ & $2.87 \pm 0.07$ & $2.88 \pm 0.03$ & 82 & $\mathrm{~Pb}$ & $1.75 \pm 0.10$ & $2.04 \pm 0.03$ \\
\hline 39 & $\mathrm{Y}$ & $2.21 \pm 0.05$ & $2.17 \pm 0.04$ & 83 & $\mathrm{Bi}$ & & $0.65 \pm 0.04$ \\
\hline 40 & $\mathrm{Zr}$ & $2.58 \pm 0.04$ & $2.53 \pm 0.04$ & 90 & $\mathrm{Th}$ & $0.02 \pm 0.10$ & $0.06 \pm 0.03$ \\
\hline 41 & $\mathrm{Nb}$ & $1.46 \pm 0.04$ & $1.41 \pm 0.04$ & 92 & $\mathrm{U}$ & & $-0.54 \pm 0.03$ \\
\hline 42 & Mo & $1.88 \pm 0.08$ & $1.94 \pm 0.04$ & & & & \\
\hline
\end{tabular}


Table 2: The derived photospheric solar $\mathrm{C}, \mathrm{N}$ and $\mathrm{O}$ abundances as indicated by various atomic and molecular species and a 3D hydrodynamical model of the solar atmosphere (Asplund et al. 2009a. d, Sauval et al. 2009). The corresponding results obtained with the temporally and spatially (over equal optical depth surfaces) averaged 3D model (denoted $\langle 3 \mathrm{D}\rangle$ ), the 1D theoretical MARCS model (Gustafsson et al. 2008) and the 1D semi-empirical model of Holweger \& Müller (1974) are also given for comparison purposes. The errors given here are only the standard deviation $(\sigma)$ for each type of transition. For the atomic transitions, departures from LTE have been considered.

\begin{tabular}{|c|c|c|c|c|}
\hline \multirow[t]{2}{*}{ lines } & \multicolumn{4}{|c|}{$\log \epsilon_{\mathrm{C}, \mathrm{N}, \mathrm{O}}$} \\
\hline & $3 \mathrm{D}$ & $<3 \mathrm{D}>$ & HM & MARCS \\
\hline$[\mathrm{C} \mathrm{I}]$ & 8.41 & 8.40 & 8.41 & 8.38 \\
\hline $\mathrm{C}_{\mathrm{I}}$ & $8.42 \pm 0.05$ & $8.47 \pm 0.04$ & $8.45 \pm 0.04$ & $8.39 \pm 0.04$ \\
\hline $\mathrm{CH} \Delta v=1$ & $8.44 \pm 0.04$ & $8.44 \pm 0.04$ & $8.53 \pm 0.04$ & $8.44 \pm 0.04$ \\
\hline CH A-X & $8.43 \pm 0.03$ & $8.42 \pm 0.03$ & $8.51 \pm 0.03$ & $8.40 \pm 0.03$ \\
\hline $\mathrm{C}_{2}$ Swan & $8.46 \pm 0.03$ & $8.46 \pm 0.03$ & $8.51 \pm 0.03$ & $8.46 \pm 0.03$ \\
\hline N I & $7.78 \pm 0.04$ & $7.89 \pm 0.04$ & $7.88 \pm 0.04$ & $7.78 \pm 0.04$ \\
\hline $\mathrm{NH} \Delta v=0$ & $7.83 \pm 0.03$ & $7.94 \pm 0.02$ & $8.02 \pm 0.02$ & $7.97 \pm 0.02$ \\
\hline $\mathrm{NH} \Delta v=1$ & $7.88 \pm 0.03$ & $7.91 \pm 0.03$ & $8.01 \pm 0.03$ & $7.91 \pm 0.03$ \\
\hline$[\mathrm{O} \mathrm{I}]$ & $8.70 \pm 0.05$ & $8.70 \pm 0.05$ & $8.73 \pm 0.05$ & $8.69 \pm 0.05$ \\
\hline O I & $8.69 \pm 0.05$ & $8.73 \pm 0.05$ & $8.69 \pm 0.05$ & $8.62 \pm 0.05$ \\
\hline $\mathrm{OH} \Delta v=0$ & $8.69 \pm 0.03$ & $8.75 \pm 0.03$ & $8.83 \pm 0.03$ & $8.78 \pm 0.03$ \\
\hline $\mathrm{OH} \Delta v=1$ & $8.69 \pm 0.03$ & $8.74 \pm 0.03$ & $8.86 \pm 0.03$ & $8.75 \pm 0.03$ \\
\hline
\end{tabular}


Table 3: Representative isotopic abundance fractions in the solar system. Most of the isotopic values are taken from Rosman \& Taylor (1998) with updates for some elements, as discussed in Sect. 3.10.

\begin{tabular}{|c|c|c|c|c|c|c|c|c|c|c|c|c|c|}
\hline$Z$ & $A$ & $\%$ & $Z$ & $A$ & $\%$ & $Z$ & $A$ & $\%$ & $Z$ & $A$ & $\%$ & $Z$ & $A \quad \%$ \\
\hline \multirow[t]{3}{*}{$\mathrm{H}$} & 1 & 99.998 & $\mathrm{~S}$ & 32 & 94.93 & $\mathrm{Fe}$ & 57 & 2.119 & $\mathrm{Kr}$ & 82 & 11.655 & $\mathrm{Pd}$ & 10522.33 \\
\hline & 2 & 0.002 & & 33 & 0.76 & & 58 & 0.282 & & 83 & 11.546 & & 10627.33 \\
\hline & & & & 34 & 4.29 & & & & & 84 & 56.903 & & 10826.46 \\
\hline \multirow[t]{3}{*}{$\mathrm{He}$} & 3 & 0.0166 & & 36 & 0.02 & $\mathrm{Co}$ & 59 & 100.0 & & 86 & 17.208 & & 11011.72 \\
\hline & 4 & 99.9834 & & & & & & & & & & & \\
\hline & & & $\mathrm{Cl}$ & 35 & 75.78 & $\mathrm{Ni}$ & 58 & 68.0769 & $\mathrm{Rb}$ & 85 & 70.844 & $\mathrm{Ag}$ & 10751.839 \\
\hline \multirow[t]{3}{*}{$\mathrm{Li}$} & 6 & 7.59 & & 37 & 24.22 & & 60 & 26.2231 & & 87 & 29.156 & & 10948.161 \\
\hline & 7 & 92.41 & & & & & 61 & 1.1399 & & & & & \\
\hline & & & $\mathrm{Ar}$ & 36 & 84.5946 & & 62 & 3.6345 & $\mathrm{Sr}$ & 84 & 0.5580 & $\mathrm{Cd}$ & 1061.25 \\
\hline \multirow[t]{2}{*}{$\mathrm{Be}$} & 9 & 100.0 & & 38 & 15.3808 & & 64 & 0.9256 & & 86 & 9.8678 & & 1080.89 \\
\hline & & & & 40 & 0.0246 & & & & & 87 & 6.8961 & & 11012.49 \\
\hline \multirow[t]{3}{*}{ B } & 10 & 19.9 & & & & $\mathrm{Cu}$ & 63 & 69.17 & & 88 & 82.6781 & & 11112.80 \\
\hline & 11 & 80.1 & $\mathrm{~K}$ & 39 & 93.132 & & 65 & 30.83 & & & & & 11224.13 \\
\hline & & & & 40 & 0.147 & & & & $\mathrm{Y}$ & 89 & 100.0 & & 11312.22 \\
\hline \multirow[t]{3}{*}{$\mathrm{C}$} & 12 & 98.8938 & & 41 & 6.721 & $\mathrm{Zn}$ & 64 & 48.63 & & & & & 11428.73 \\
\hline & 13 & 1.1062 & & & & & 66 & 27.90 & $\mathrm{Zr}$ & 90 & 51.45 & & 1167.49 \\
\hline & & & $\mathrm{Ca}$ & 40 & 96.941 & & 67 & 4.10 & & 91 & 11.22 & & \\
\hline \multirow[t]{3}{*}{$\mathrm{N}$} & 14 & 99.771 & & 42 & 0.647 & & 68 & 18.75 & & 92 & 17.15 & In & 1134.29 \\
\hline & 15 & 0.229 & & 43 & 0.135 & & 70 & 0.62 & & 94 & 17.38 & & 11595.71 \\
\hline & & & & 44 & 2.086 & & & & & 96 & 2.80 & & \\
\hline \multirow[t]{4}{*}{$\mathrm{O}$} & 16 & 99.7621 & & 46 & 0.004 & $\mathrm{Ga}$ & 69 & 60.108 & & & & Sn & 1120.97 \\
\hline & 17 & 0.0379 & & 48 & 0.187 & & 71 & 39.892 & $\mathrm{Nb}$ & 93 & 100.0 & & 1140.66 \\
\hline & 18 & 0.2000 & & & & & & & & & & & 1150.34 \\
\hline & & & $\mathrm{Sc}$ & 45 & 100.0 & $\mathrm{Ge}$ & 70 & 20.84 & Mo & 92 & 14.525 & & 11614.54 \\
\hline \multirow[t]{2}{*}{$\mathrm{F}$} & 19 & 100.0 & & & & & 72 & 27.54 & & 94 & 9.151 & & 1177.68 \\
\hline & & & $\mathrm{Ti}$ & 46 & 8.25 & & 73 & 7.73 & & 95 & 15.838 & & 11824.22 \\
\hline \multirow[t]{4}{*}{$\mathrm{Ne}$} & 20 & 92.9431 & & 47 & 7.44 & & 74 & 36.28 & & 96 & 16.672 & & 1198.59 \\
\hline & 21 & 0.2228 & & 48 & 73.72 & & 76 & 7.61 & & 97 & 9.599 & & 12032.58 \\
\hline & 22 & 6.8341 & & 49 & 5.41 & & & & & 98 & 24.391 & & 1224.63 \\
\hline & & & & 50 & 5.18 & As & 75 & 100.0 & & 100 & 9.824 & & 1245.79 \\
\hline \multirow[t]{2}{*}{$\mathrm{Na}$} & 23 & 100.0 & & & & & & & & & & & \\
\hline & & & $\mathrm{V}$ & 50 & 0.250 & $\mathrm{Se}$ & 74 & 0.89 & $\mathrm{Ru}$ & 96 & 5.54 & $\mathrm{Sb}$ & 12157.21 \\
\hline \multirow[t]{4}{*}{$\mathrm{Mg}$} & 24 & 78.99 & & 51 & 99.750 & & 76 & 9.37 & & 98 & 1.87 & & 12342.79 \\
\hline & 25 & 10.00 & & & & & 77 & 7.63 & & 99 & 12.76 & & \\
\hline & 26 & 11.01 & $\mathrm{Cr}$ & 50 & 4.345 & & 78 & 23.77 & & 100 & 12.60 & $\mathrm{Te}$ & 1200.09 \\
\hline & & & & 52 & 83.789 & & 80 & 49.61 & & 101 & 17.06 & & 1222.55 \\
\hline \multirow[t]{2}{*}{$\mathrm{Al}$} & 27 & 100.0 & & 53 & 9.501 & & 82 & 8.73 & & 102 & 31.55 & & 1230.89 \\
\hline & & & & 54 & 2.365 & & & & & 104 & 118.62 & & 1244.74 \\
\hline \multirow[t]{4}{*}{$\mathrm{Si}$} & 28 & 92.2297 & & & & $\mathrm{Br}$ & 79 & 50.69 & & & & & 1257.07 \\
\hline & 29 & 4.6832 & $\mathrm{Mn}$ & 55 & 100.0 & & 81 & 49.31 & $\mathrm{Rh}$ & 103 & 3100.0 & & 12618.84 \\
\hline & 30 & 3.0872 & & & & & & & & & & & 12831.74 \\
\hline & & & $\mathrm{Fe}$ & 54 & 5.845 & $\mathrm{Kr}$ & 78 & 0.362 & $\mathrm{Pd}$ & 102 & 1.02 & & 13034.08 \\
\hline $\mathrm{P}$ & 31 & 100.0 & & 56 & 91.754 & & 80 & 2.326 & & 104 & 11.14 & & \\
\hline
\end{tabular}




\begin{tabular}{|c|c|c|c|c|c|c|c|c|}
\hline$Z$ & $A \%$ & $Z A \%$ & $Z$ & $A \%$ & $Z$ & $A \%$ & $Z$ & $A \%$ \\
\hline I & 127100.0 & $\begin{array}{r}\mathrm{Nd} 14227.044 \\
14312.023\end{array}$ & & $\begin{array}{l}1602.329 \\
16118.889\end{array}$ & $\mathrm{Hf}$ & $\begin{array}{l}17827.297 \\
17913.629\end{array}$ & $\mathrm{Pt}$ & $\begin{array}{l}19625.242 \\
1987.163\end{array}$ \\
\hline $\mathrm{Xe}$ & 1240.122 & 14423.729 & & 16225.475 & & 18035.100 & & \\
\hline & 1260.108 & 1458.763 & & 16324.896 & & & $\mathrm{Au}$ & 197100.0 \\
\hline & 1282.188 & 14617.130 & & 16428.260 & $\mathrm{Ta}$ & 1800.012 & & \\
\hline & 12927.255 & 1485.716 & & & & 18199.988 & $\mathrm{Hg}$ & 1960.15 \\
\hline & 1304.376 & 1505.596 & Ho & 165100.0 & & & & 1989.97 \\
\hline & 13121.693 & & & & W & 1800.12 & & 19916.87 \\
\hline & 13226.514 & Sm 1443.07 & Er & 1620.139 & & 18226.50 & & 20023.10 \\
\hline & 1349.790 & 14714.99 & & 1641.601 & & 18314.31 & & 20113.18 \\
\hline & 1367.954 & 14811.24 & & 16633.503 & & 18430.64 & & 20229.86 \\
\hline & & 14913.82 & & 16722.869 & & 18628.43 & & 2046.87 \\
\hline Cs & 133100.0 & 1507.38 & & 16826.978 & & & & \\
\hline & & 15226.75 & & 17014.910 & $\operatorname{Re}$ & 18535.662 & $\mathrm{Tl}$ & 20329.524 \\
\hline $\mathrm{Ba}$ & 1300.106 & 15422.75 & & & & 18764.338 & & 20570.476 \\
\hline & 1320.101 & & $\operatorname{Tm}$ & n169100.0 & & & & \\
\hline & 1342.417 & $\mathrm{Eu} 15147.81$ & & & Os & 1840.020 & $\mathrm{~Pb}$ & 2041.997 \\
\hline & 1356.592 & 15352.19 & & 1680.12 & & 1861.598 & & 20618.582 \\
\hline & 1367.854 & & & 1702.98 & & 1871.271 & & 20720.563 \\
\hline & 13711.232 & Gd 1520.20 & & 17114.09 & & 18813.337 & & 20858.858 \\
\hline & 13871.698 & 1542.18 & & 17221.69 & & 18916.261 & & \\
\hline & & 15514.80 & & 17316.10 & & 19026.444 & $\mathrm{Bi}$ & 209100.0 \\
\hline $\mathrm{La}$ & 1380.091 & 15620.47 & & 17432.03 & & 19241.070 & & \\
\hline & 13999.909 & 15715.65 & & 17613.00 & & & $\mathrm{Tl}$ & 232100.0 \\
\hline $\mathrm{Ce}$ & 1360.185 & $\begin{array}{l}15824.84 \\
16021.86\end{array}$ & $\mathrm{Lu}$ & 17597.1795 & Ir & $\begin{array}{l}19137.3 \\
19362.7\end{array}$ & $\mathrm{U}$ & 2340.002 \\
\hline & 1380.251 & & & 1762.8205 & & & & 23524.286 \\
\hline & 14088.450 & Tb 159100.0 & & & $\mathrm{Pt}$ & 1900.014 & & 23875.712 \\
\hline & 14211.114 & & $\mathrm{Hf}$ & 1740.162 & & 1920.782 & & \\
\hline & & Dy 1560.056 & & 1765.206 & & 19432.967 & & \\
\hline $\operatorname{Pr}$ & 141100.0 & 1580.095 & & 17718.606 & & 19533.832 & & \\
\hline
\end{tabular}


Table 4: The mass fractions of hydrogen (X), helium (Y) and metals (Z) for a number of widely-used compilations of the solar chemical composition.

\begin{tabular}{|c|c|c|c|c|}
\hline Source & $X$ & $Y$ & $Z$ & $Z / X$ \\
\hline \multicolumn{5}{|l|}{ Present-day photosphere: } \\
\hline Anders \& Grevesse $(1989)^{\mathrm{a}}$ & 0.7314 & 0.2485 & 0.0201 & 0.0274 \\
\hline Grevesse \& Noels $(1993)^{\mathrm{a}}$ & 0.7336 & 0.2485 & 0.0179 & 0.0244 \\
\hline Grevesse \& Sauval (1998) & 0.7345 & 0.2485 & 0.0169 & 0.0231 \\
\hline Lodders (2003) & 0.7491 & 0.2377 & 0.0133 & 0.0177 \\
\hline Asplund. Grevesse \& Sauval (2005) & 0.7392 & 0.2485 & 0.0122 & 0.0165 \\
\hline Lodders. Palme \& Gail (2009) & 0.7390 & 0.2469 & 0.0141 & 0.0191 \\
\hline Present work & 0.7381 & 0.2485 & 0.0134 & 0.0181 \\
\hline \multicolumn{5}{|l|}{ Proto-solar: } \\
\hline Anders \& Grevesse (1989) & 0.7096 & 0.2691 & 0.0213 & 0.0301 \\
\hline Grevesse \& Noels (1993) & 0.7112 & 0.2697 & 0.0190 & 0.0268 \\
\hline Grevesse \& Sauval (1998) & 0.7120 & 0.2701 & 0.0180 & 0.0253 \\
\hline Lodders (2003) & 0.7111 & 0.2741 & 0.0149 & 0.0210 \\
\hline Asplund. Grevesse \& Sauval (2005) & 0.7166 & 0.2704 & 0.0130 & 0.0181 \\
\hline Lodders. Palme \& Gail (2009) & 0.7112 & 0.2735 & 0.0153 & 0.0215 \\
\hline Present work & 0.7154 & 0.2703 & 0.0142 & 0.0199 \\
\hline
\end{tabular}

a The He abundances given in Anders \& Grevesse (1989) and Grevesse \& Noels (1993) have here been replaced with the current best estimate from helioseismology (Sect. 3.9).

Table 5: Comparison of the proto-solar abundances from the present work and Grevesse \& Sauval (1998) with those in nearby B stars and H II regions. The solar values given here include the effects of diffusion (Turcotte \& Wimmer-Schweingruber 2002) as discussed in Sect. 3.11. The H II numbers include the estimated elemental fractions tied up in dust; the dust corrections for $\mathrm{Mg}$, Si and $\mathrm{Fe}$ are very large and thus too uncertain to provide meaningful values here. Also given in the last column is the predicted Galactic chemical enrichment (GCE) over the past 4.56 Gyr.

\begin{tabular}{|c|c|c|c|c|c|}
\hline Elem. & Sun $^{\mathrm{a}}$ & Sun $^{\mathrm{b}}$ & B stars ${ }^{\mathrm{c}}$ & $\mathrm{H} \mathrm{II}^{\mathrm{d}}$ & $\mathrm{GCE}^{\mathrm{e}}$ \\
\hline $\mathrm{He}$ & $10.98 \pm 0.01$ & $10.98 \pm 0.01$ & $10.98 \pm 0.02$ & $10.96 \pm 0.01$ & 0.01 \\
\hline $\mathrm{C}$ & $8.56 \pm 0.06$ & $8.47 \pm 0.05$ & $8.35 \pm 0.03$ & $8.66 \pm 0.06$ & 0.06 \\
\hline $\mathrm{N}$ & $7.96 \pm 0.06$ & $7.87 \pm 0.05$ & $7.76 \pm 0.05$ & $7.85 \pm 0.06$ & 0.08 \\
\hline $\mathrm{O}$ & $8.87 \pm 0.06$ & $8.73 \pm 0.05$ & $8.76 \pm 0.03$ & $8.80 \pm 0.04$ & 0.04 \\
\hline $\mathrm{Ne}$ & $8.12 \pm 0.06$ & $7.97 \pm 0.10$ & $8.08 \pm 0.03$ & $8.00 \pm 0.08$ & 0.04 \\
\hline $\mathrm{Mg}$ & $7.62 \pm 0.05$ & $7.64 \pm 0.04$ & $7.56 \pm 0.05$ & & 0.04 \\
\hline $\mathrm{Si}$ & $7.59 \pm 0.05$ & $7.55 \pm 0.04$ & $7.50 \pm 0.02$ & & 0.08 \\
\hline $\mathrm{S}$ & $7.37 \pm 0.11$ & $7.16 \pm 0.03$ & $7.21 \pm 0.13$ & $7.30 \pm 0.04$ & 0.09 \\
\hline $\mathrm{Ar}$ & $6.44 \pm 0.06$ & $6.44 \pm 0.13$ & $6.66 \pm 0.06$ & $6.62 \pm 0.06$ & \\
\hline $\mathrm{Fe}$ & $7.55 \pm 0.05$ & $7.54 \pm 0.04$ & $7.44 \pm 0.04$ & & 0.14 \\
\hline G & e \& Sauval & \begin{tabular}{l|l}
$1998)$ & $\mathrm{b}$ \\
$06)$ & Lanz
\end{tabular} & sent work & $\begin{array}{l}\text { Przvbilla. } \\
\text { teban et al }\end{array}$ & va \& \\
\hline
\end{tabular}




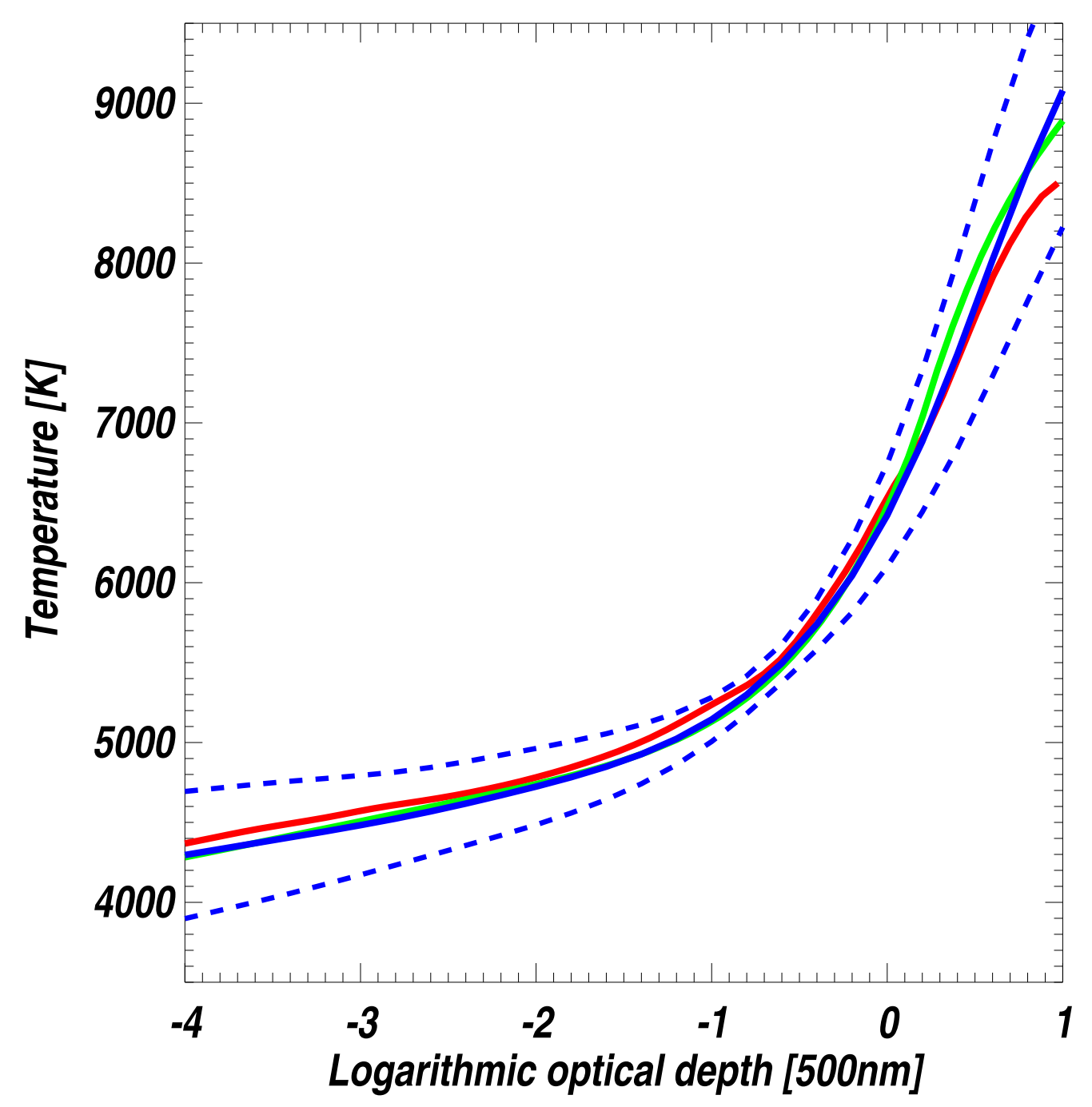

Figure 1: The mean temperature structure of the 3D hydrodynamical model of Trampedach et al. (2009) is shown as a function of optical depth at $500 \mathrm{~nm}$ (blue solid line). The blue dashed lines correspond to the spatial and temporal rms variations of the 3D model, while the red and green curves denote the 1D semi-empirical Holweger \& Müller (1974) and the 1D theoretical MARCS (Gustafsson et al. 2008) model atmospheres, respectively. 


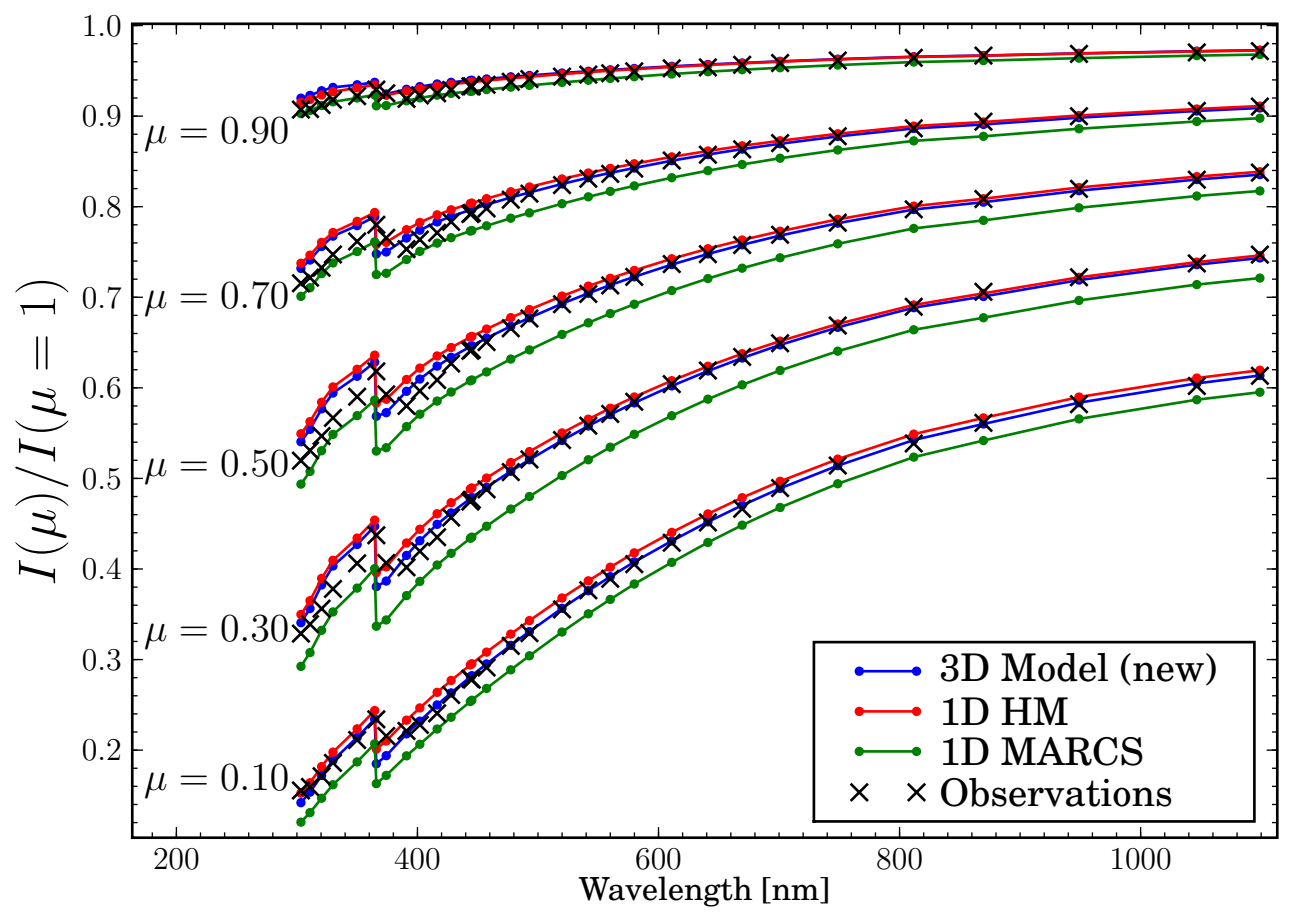

Figure 2: Comparison of the predicted continuum center-to-limb variation as a function of wavelength for different solar model atmospheres against observations (Neckel \& Labs 1994). The results for five different viewing angles $\mu$ are shown from near disk center $(\mu=0.9)$ to close to the limb $(\mu=0.1)$. All intensities are normalized to the corresponding disk-center intensities. The 3D hydrodynamical model (Trampedach et al. 2009) outperforms even the Holweger \& Müller (1974) semi-empirical model, which was designed to satisfy this diagnostic (Pereira et al. 2009). As for all 1D theoretical model atmospheres, the MARCs model has a too steep temperature gradient, which manifests itself in a poor agreement with the center-to-limb variation. 


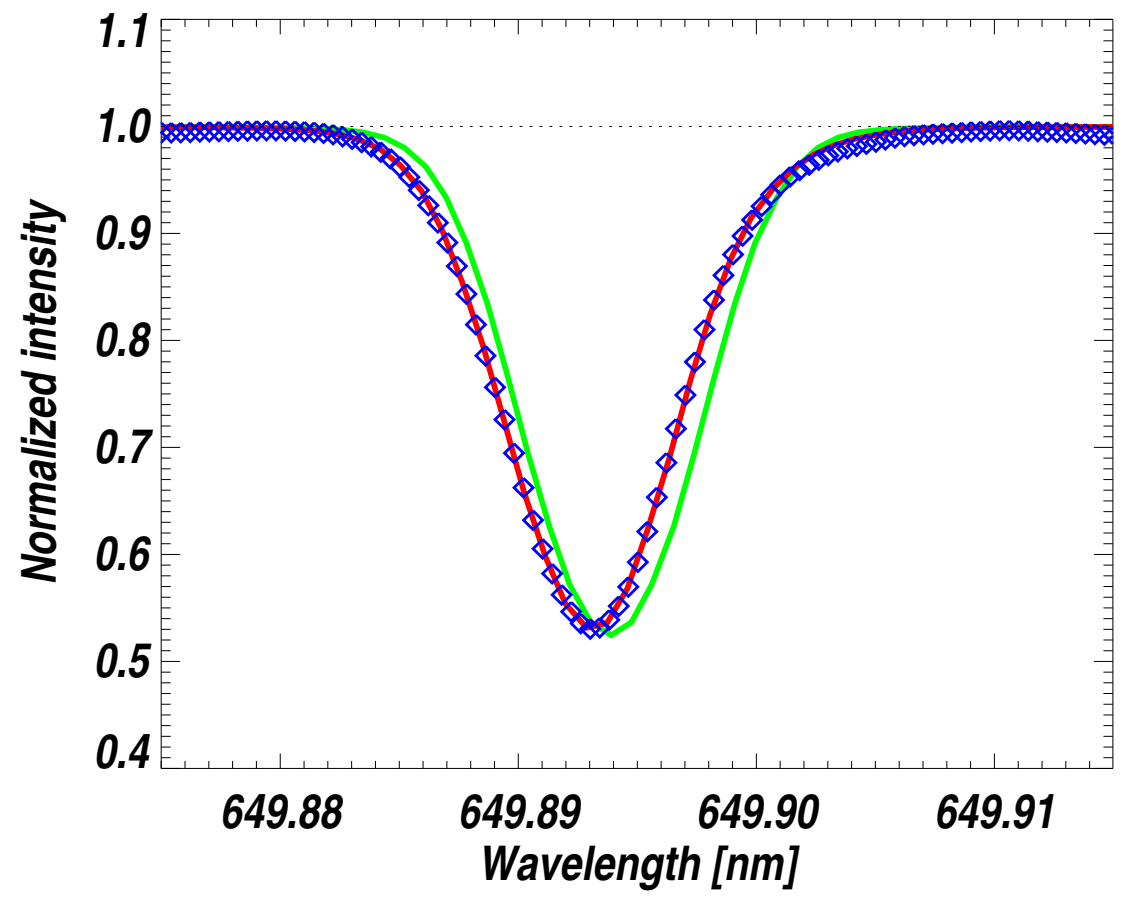

Figure 3: The predicted spectral line profile of a typical Fe I line from the 3D hydrodynamical solar model (red solid line) compared with the observations (blue rhombs). The agreement is clearly very satisfactory, which is the result of the Doppler shifts arising from the self-consistently computed convective motions that broaden, shift and skew the theoretical profile. For comparison purposes also the predicted profile from a 1D model atmosphere (here Holweger \& Müller 1974) is shown; the 1D profile has been computed with a microturbulence of $1 \mathrm{~km} \mathrm{~s}^{-1}$ and a tuned macroturbulence to obtain the right overall linewidth. Note that even with these two free parameters the $1 \mathrm{D}$ profile can neither predict the shift nor the asymmetry of the line. 


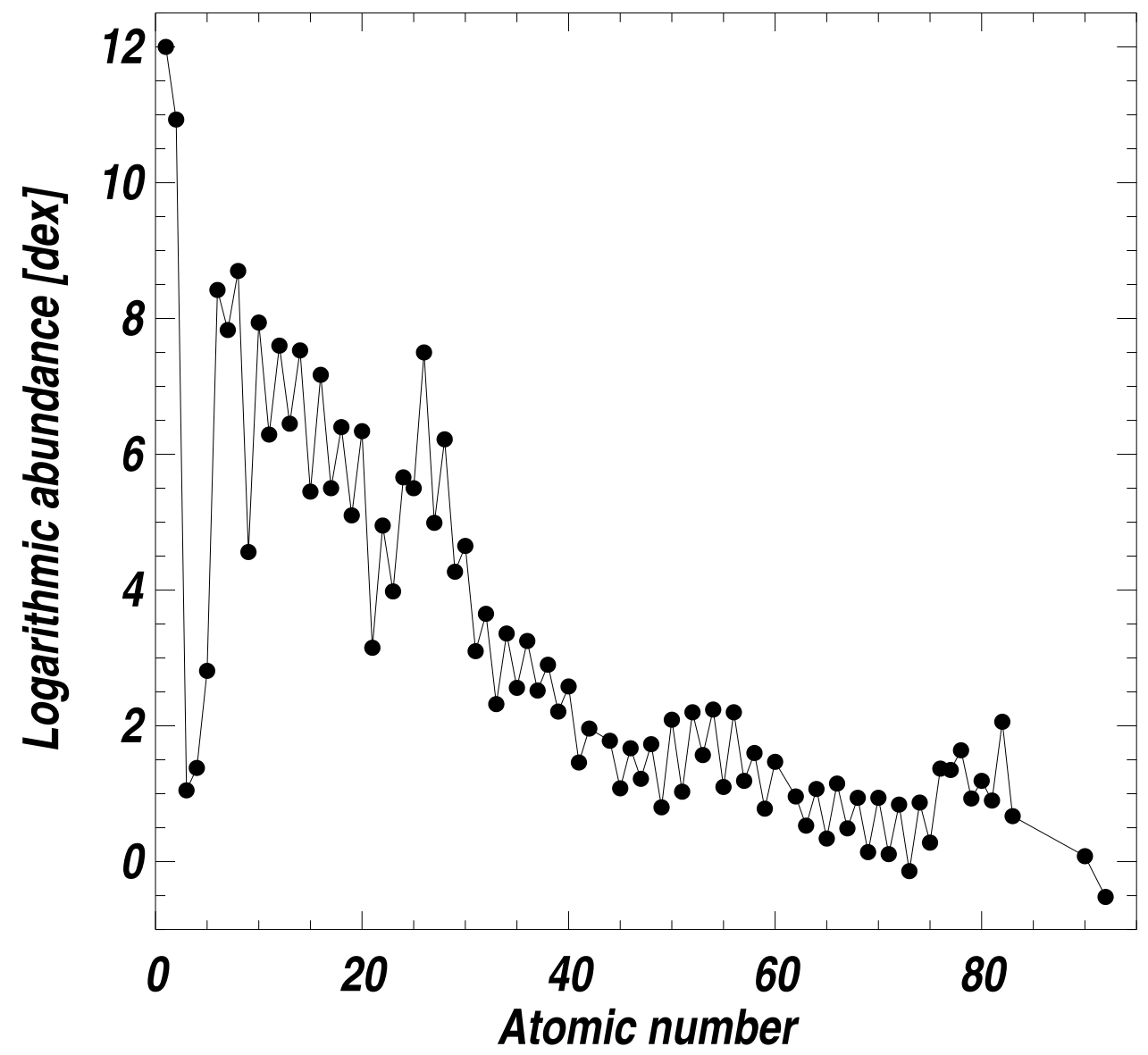

Figure 4: The present-day solar photospheric elemental abundances as a function of atomic number. As throughout this article, the logarithmic abundance of hydrogen is defined to be $\log \epsilon_{\mathrm{H}}=12.0$. 


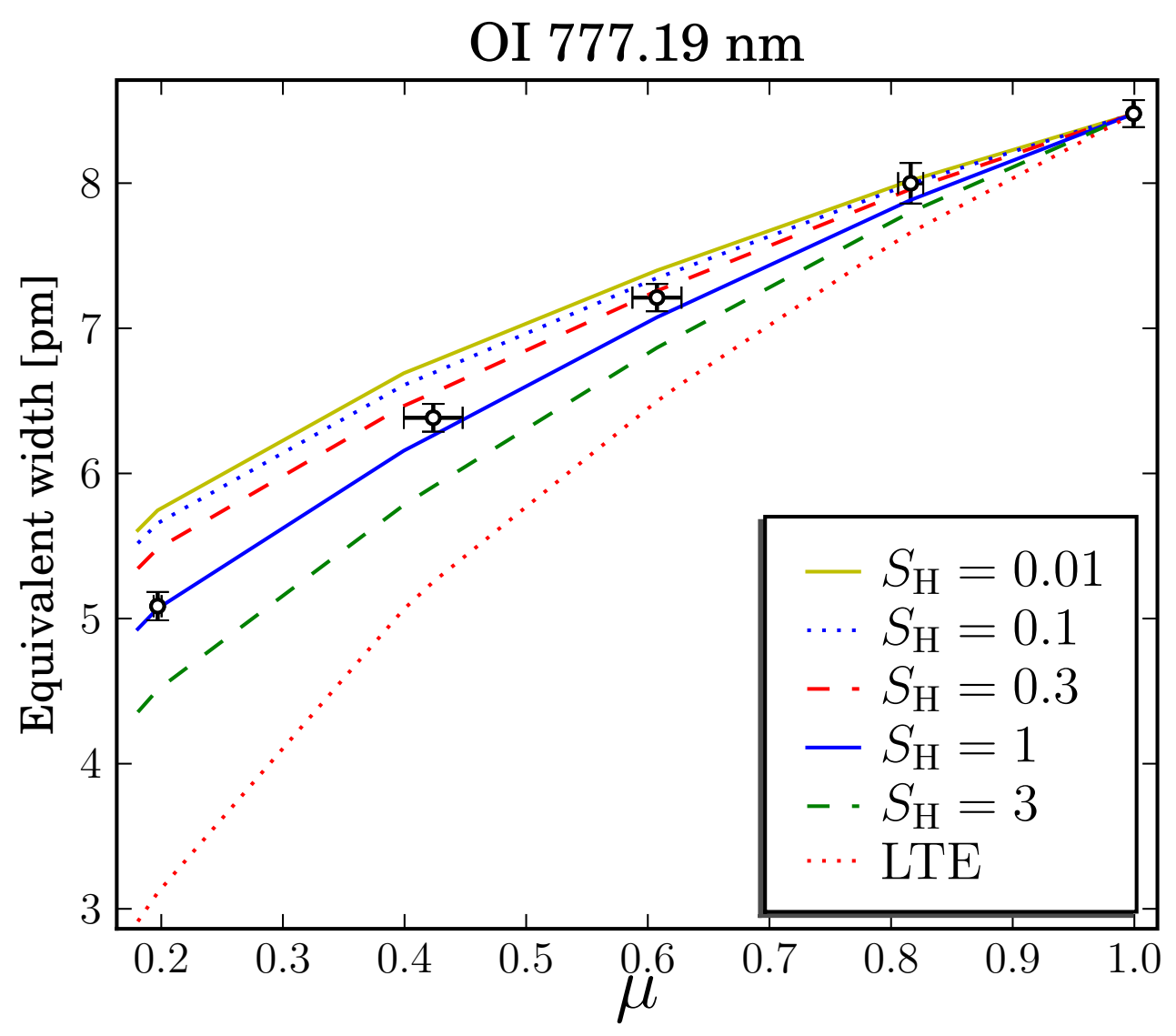

Figure 5: Comparison of the observed (open circles with error bars) and predicted center-to-limb variation of the equivalent width of the O I $777.19 \mathrm{~nm}$ triplet line as a function of viewing angle $(\mu=1$ corresponds to disk center and 0 to the limb). The theoretical curves are the results of full 3D non-LTE line formation calculations with different adopted efficiency for the poorly known inelastic $\mathrm{H}$ collisions; the $\mathrm{O}$ abundances have been normalized to fit the disk-center line strengths in each case. The curves are labelled with the scaling factor used to multiply the classical Drawin (1968) formula. The solar observations thus seem to suggest that this recipe is a rather accurate representation for O I (Pereira, Asplund \& Kiselman 2009). 

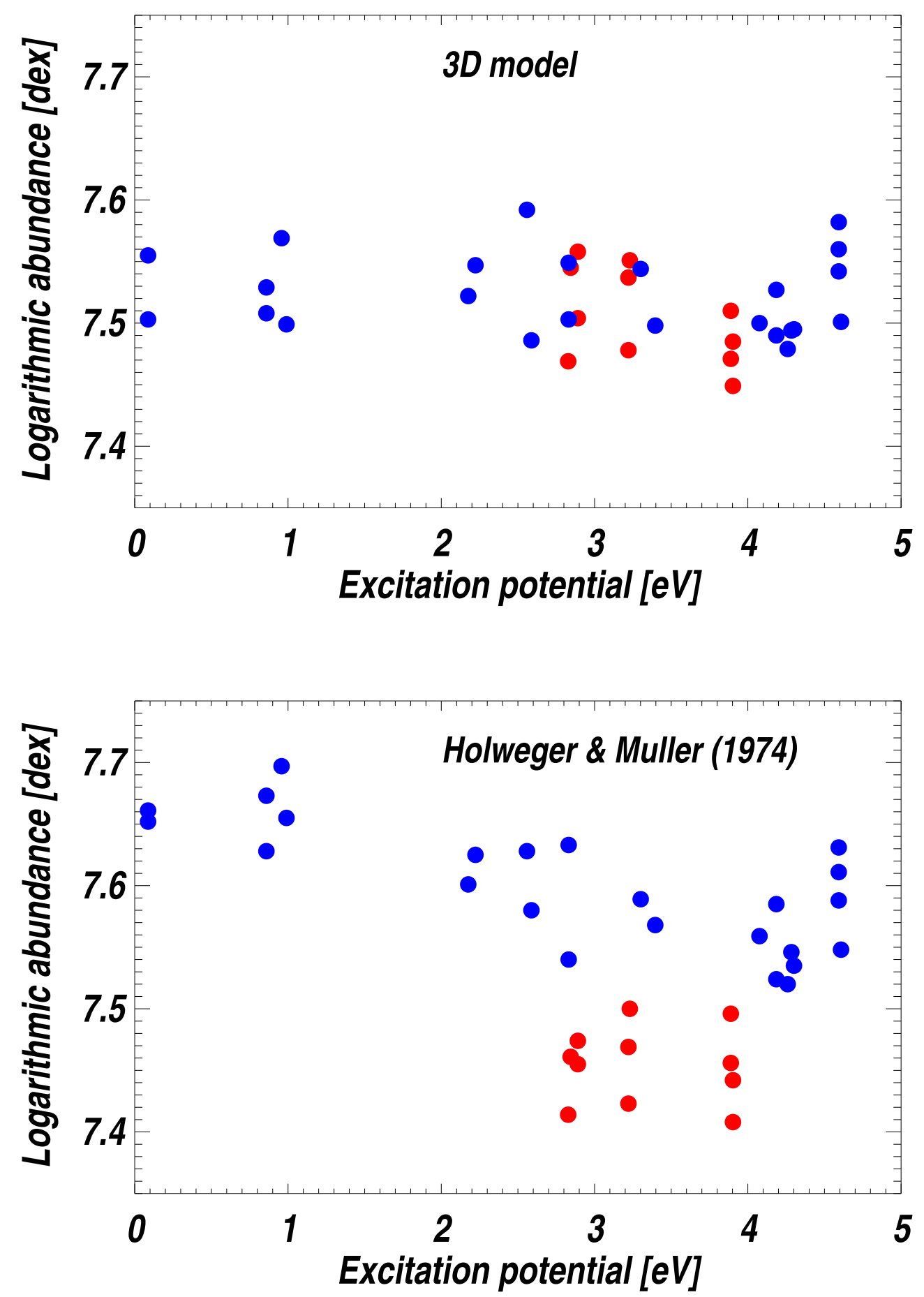

Figure 6: Upper panel: The derived Fe I (blue circles) and Fe II (red circles) abundances (Scott et al. 2009b) as a function of excitation potential of the lower level when using a 3D hydrodynamical solar model atmosphere. Lower panel: Same as above but with the 1D semi-empirical Holweger \& Müller (1974) model. Note the offset between Fe I and Fe II and the trend with excitation potential for the Fe I lines, neither of which are present when employing the 3D model. 


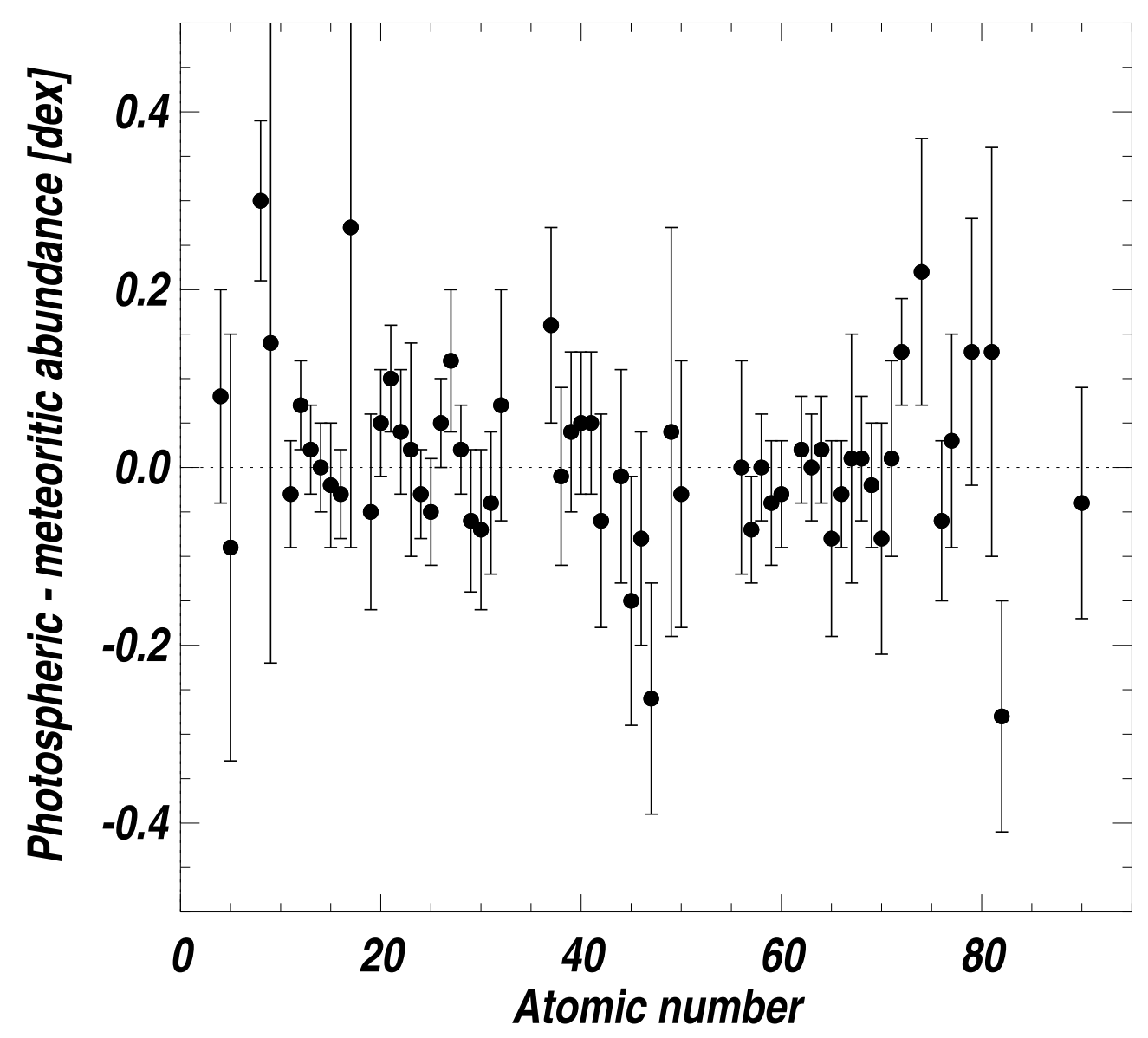

Figure 7: Difference between the logarithmic abundances determined from the solar photosphere and the CI carbonaceous chondrites as a function of atomic number. With a few exceptions the agreement is excellent. Note that due to depletion in the Sun and meteorites, the data points for Li, C, N and the noble gases fall outside the range of the figure. 


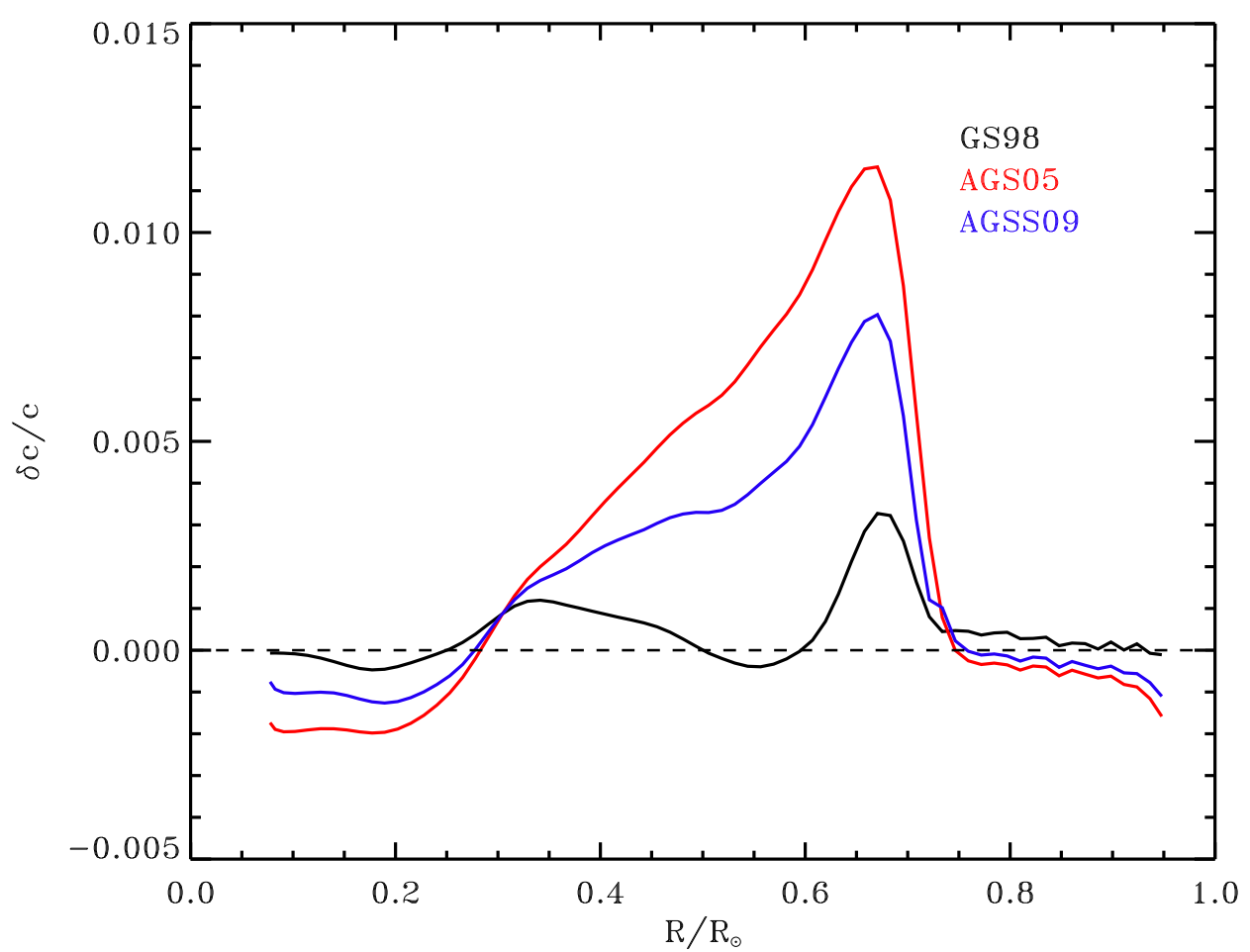

Figure 8: The differences between the helioseismic and predicted sound speeds as a function of depth (Serenelli et al. 2009). The standard solar models shown here only differ in the assumed chemical compositions: Grevesse \& Sauval (1998) (black line, here denoted GS98), Asplund, Grevesse \& Sauval (2005) (red line, AGS05) and the present work (blue line, AGSS09). Each model has independently been calibrated to achieve the correct solar luminosity, temperature and age. The base of the convection zone is at $R=0.71 \mathrm{R}_{\odot}$, which is also where the discrepancy starts in earnest in all three cases. 